\title{
Complutum
}

ISSN: 1131-6993

\section{Manipulación de restos humanos en el Bronce Medio meseteño: el fragmento fronto-facial hallado en el yacimiento de La Huelga (Dueñas, Palencia)}

\author{
Ángel Esparza Arroyoํㄱ Javier Velasco Vázquez²; Alejandra Sánchez Polo
}

Recibido: 27 de marzo de 2020 / Aceptado: 22 de junio de 2020

Resumen. La investigación sobre restos humanos prehistóricos, tradicionalmente centrada en las sepulturas y enterramientos de esqueletos completos, relegó el estudio de los restos secundarios y aislados, cuya gran importancia en relación con prácticas mortuorias complejas se ha ido revelando, por contra, en la bibliografía antropológica y bioarqueológica. En el caso de los yacimientos de la Edad del Bronce del interior peninsular, la creciente presencia de 'huesos sueltos' reclama similar consideración, y aquí se acomete el estudio de un fragmento craneal (frontal y cara) de un subadulto, datado por radiocarbono en el intervalo 1660-1497 cal BC, correspondiente a la fase Formativa de la cultura de Cogotas I, y en el que se han detectado impactos y fracturas que evidencian un tratamiento peri mortem que debió de realizarse para separar esta parte respecto de la calvaria. Para intentar comprender su significado se analizan diversas posibilidades - p.ej., sepultura destruida, 'máscara facial' o residuo de la preparación de un elemento neurocraneal-, en relación con los comportamientos sociales que conllevan la manipulación de restos humanos, incluyendo los de fragmentación y supresión de lo individual. El avance en el conocimiento de la sociedad que realizaba estas prácticas mortuorias requiere prestar atención a su heterogénea evidencia, que incluye los restos, "sueltos" y aparentemente insignificantes como este fragmento de La Huelga.

Palabras clave. Península Ibérica; Proto-Cogotas I; bioarqueología; prácticas mortuorias; máscara facial; eliminación de la cara.

[en] Human bone manipulation in the Middle Bronze Age of the Iberian Meseta: the fronto-facial fragment found at La Huelga site (Dueñas, Palencia)

Abstract. Research on prehistoric human remains primarily focuses on tombs and burials with full-representation skeletons, relegating the study of secondary and isolated remains whose great importance for the knowledge of mortuary practices is gradually being revealed in the anthropological and bioarchaeological literature. In the case of the Bronze Age sites placed in the inland Iberian Peninsula, the increasing presence of loose bones demands similar consideration. To this end, this paper undertakes the analysis of a cranial fragment (frontal and facial skeleton) of a subadult individual. The radiocarbon analysis places the death of this individual between 1660-1497 Cal BC, linked to the formative phase of Cogotas I archaeological culture. The fragment shows chop marks and fractures that reveal a perimortem treatment to separate the frontal and the facial bones from the rest of the skull. To try to understand its meaning, different possibilities are analyzed -e.g., destroyed tomb, 'facial mask' or residue from the preparation of a neurocranial object- in relation to social behaviours involving the manipulation of human remains, including those of fragmentation and suppression of the individual. The advance in the knowledge of the society practicing this mortuary conducts requires paying attention to all its evidence, including the loose and apparently insignificant remains, even those without clear context as is the case of this fragment from La Huelga.

Keywords. Iberian Peninsula; Proto-Cogotas I; bioarchaeology; mortuary practices; skull mask; de-facing.

Sumario. 1. Introducción. 2. El hallazgo en su contexto. 3. Datación radiocarbónica. 4. Consideraciones bioantropológicas. 4.1. Caracterización y tafonomía del fragmento craneal. 4.2. Huellas antrópicas sobre el fragmento craneal. 4.3. ¿Cómo explicar las huellas antrópicas? 5. Discusión. 5.1. ¿Una sepultura destruida? 5.2. ¿Una 'máscara facial'? 5.3. ¿Un subproducto abandonado? 5.4. ¿Prácticas sociales de fragmentación y despersonalización? 6. Consideraciones finales. Agradecimientos. Bibliografía.

\footnotetext{
Grupo de Investigación Reconocido PREHUSAL. Departamento de Prehistoria, $H^{a}$ Antigua y Arqueología. Universidad de Salamanca. 37071 Salamanca. Correo-e: esparza@usal.es. https://orcid-org/0000-0002-9139-0774

2 Servicio de Patrimonio Histórico. Cabildo Insular de Gran Canaria. 35002 Las Palmas de Gran Canaria. Correo-e: jvelascov@, grancanaria.com. https://orcid.org/0000-0003-0339-3500

3 Grupo de Investigación Reconocido PREHUSAL. Departamento de Prehistoria, Ha Antigua y Arqueología. Universidad de Salamanca. 37071 Salamanca. Correo-e: asanpol@usal.es. https://orcid.org/0000-0001-6067-7724
} 
Cómo citar: Esparza Arroyo, A.; Velasco Vázquez, J.; Sánchez Polo, A. (2020). Manipulación de restos humanos en el Bronce Medio meseteño: el fragmento fronto-facial hallado en el yacimiento de La Huelga (Dueñas, Palencia). Complutum, 31 (1): 49-69.

\section{Introducción}

Desde momentos tempranos de la investigación prehistórica llamó la atención en muy distintos yacimientos, en contextos no siempre estrictamente funerarios, la presencia de ciertos restos humanos seleccionados, concretamente del esqueleto de la cabeza. La neta visibilidad de los cráneos, más o menos completos -a menudo interpretados en relación con cultos, o cargados de simbolismo, como supuesta sede del intelecto o del alma (Brück, 1995: 256-7)-, se refleja en la bibliografía mediante términos como 'captación de cabezas' (head hunting), 'máscaras faciales' (masques faciaux), 'cráneos-copa' (skull-cups), etc., frecuentes los dos primeros en la Edad del Hierro (Armitt, 2012; Boulestin y Duday, 2012:149) o ya desde el Paleolítico y Neolítico en el último caso (Bello et al., 2011; Santana et al., 2019; Marginedas et al., 2020), sin olvidar las abundantes y casi intemporales 'rodajas' (rondelles), tenidas por amuletos y a veces asociadas a trepanación (Camps-Fabrer, 1993: 79-87; Georgieva y Russeva, 2016).

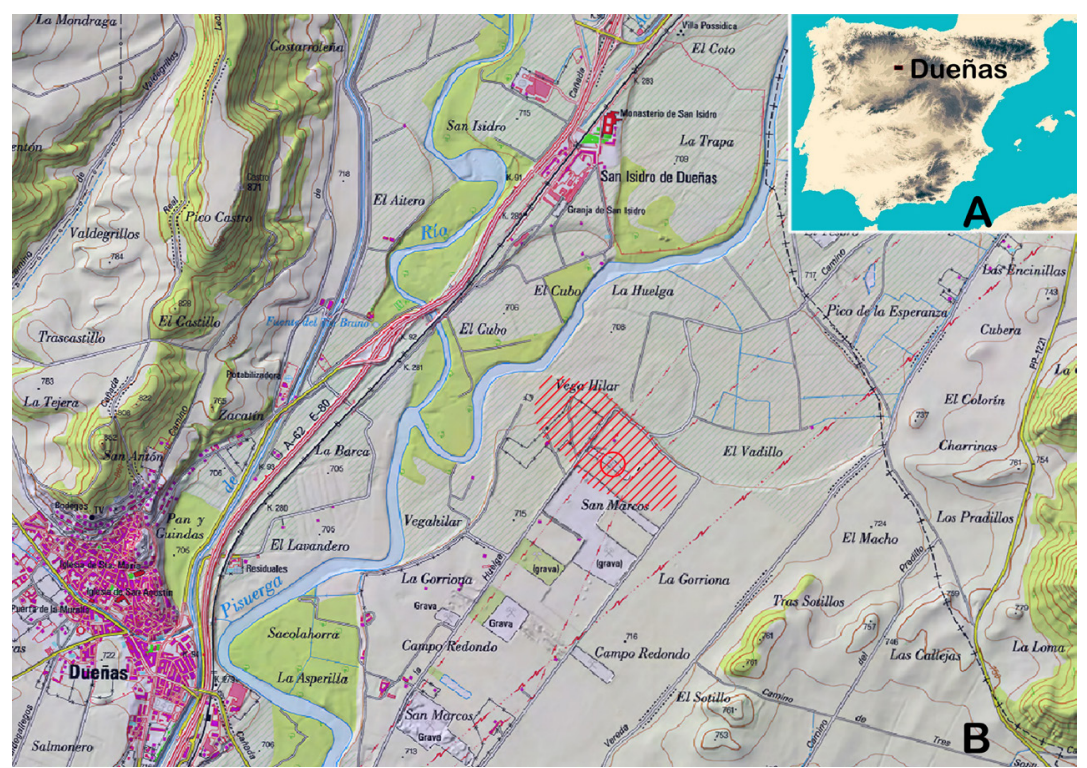

Figura 1. Localización de Dueñas en el mapa de la Península Ibérica (A) y en el Visor de la Infraestructura de Datos Espaciales de Castilla y León (B), con el yacimiento de

La Huelga (rayado) y la zona de aparición del fragmento fronto-facial (círculo).

En la Península Ibérica no faltarían tampoco ejemplos análogos, y en esta ocasión se presenta un hallazgo producido en el yacimiento palentino de La Huelga (Dueñas), que, como se verá, debe integrarse en el problemático mundo de la muerte de la cultura arqueológica de Cogotas I. En este ámbito, del que se ha ido conociendo un cierto número de enterramientos (Abarquero, 2005), sigue resultando sorprendente su bajo número con relación a los siete siglos de duración y la amplitud del área de dispersión: en el caso de la Submeseta Norte, se contaban hace poco (Esparza et al., 2012a: 276) 39 individuos en 28 enterramientos de 19 yacimientos. Se ha propuesto que debe de tratarse de enterramientos de excepción, reservados para quienes, por causas diversas, debían ser excluidos de la pauta principal, probablemente la exposición de cadáveres (Esparza et al., 2012b). Hay, en cambio, cada vez más restos secundarios - de naturaleza muy heterogénea, pero entre los cuales el cráneo, entero o fragmentado, aislado o acompañado por otros huesos, tiene una presencia relevante, en torno al 90 por ciento en el conjunto de la Submeseta Norte (Esparza et al., 2012a: 285)-, por lo que reclaman también estudio especializado sin el cual difícilmente podrá avanzarse en la comprensión del conjunto de las prácticas mortuorias ${ }^{4}$.

\footnotetext{
Partiendo del latín, en español se puede distinguir, como en otras lenguas románicas y también en inglés, entre mortuorio (relativo al muerto) y funerario (relativo al entierro y las
} 


\section{El hallazgo en su contexto}

A tres km. al NE de la localidad de Dueñas, el pago de La Huelga consiste en una amplia zona, sobre la segunda terraza del Pisuerga, en su orilla izquierda (Fig. 1). La intensa explotación del sitio como gravera motivó en los años 1991 y 1992 la realización de varias campañas de excavación preventiva, de las que dan cuenta los informes técnicos y algunas publicaciones (Misiego et al., 1992; Pérez Rodríguez et al., 1994). Estas intervenciones permiten captar el carácter del yacimiento - un 'campo de hoyos' de casi treinta hectáreas-, su diversidad de fases, asignables al Campaniforme Ciempozuelos y a la cultura de Cogotas I, especialmente en su fase Formativa, y su posible dinámica, con varias reocupaciones en sectores ligeramente desplazados, en parte superpuestos, que generan una 'estratigrafia horizontal'.

En la parcela 45 de La Huelga se efectuó en agosto de 1992 una de esas campañas de excavación relacionadas con la gravera. En las parcelas aledañas avanzaba la pala excavadora, dando lugar a la aparición de abundantes fragmentos cerámicos que eran recogidos por los arqueólogos, quienes a menudo inspeccionaban cuidadosamente las zonas removidas por la máquina. De este modo, “....en una de las frecuentes visitas al yacimiento después de esta excavación, en las escombreras de la gravera, se recuperó la parte frontal de un cráneo humano, de edad juvenil, que evidencia la existencia de una inhumación en el yacimiento" (Strato, 1992: 47), insistiéndose en que el hallazgo se produjo "en una de las escombreras de la gravera, muy cercana a la zona donde hemos excavado" (ibid.: 53) En un artículo posterior se dará a conocer también la aparición, muy próxima al lugar del cráneo, de un fragmento de puñal metálico de sección hexagonal (Pérez Rodríguez et al., 1992: 23) que pudiera relacionarse con los fragmentos cerámicos de estilo Ciempozuelos. Por ello, los investigadores dejaron abiertas las cuestiones del hallazgo craneal: "¿Se tratará de un inhumado campaniforme? ¿La inhumación sería en fosa, y adscribible a los momentos protocogotas del yacimiento?"' (Strato, 1992: 53).

Resolver tales interrogantes es uno de los objetivos del presente trabajo, en el que cabe distinguir la cuestión de la cronología, más fácil de resolver gracias al radiocarbono, y la de la interpretación del hallazgo, cuyo carácter de enterramiento no es, ni mucho menos, seguro.

\section{Datación radiocarbónica}

Las condiciones del hallazgo craneal y la compleja ocupación del yacimiento, hacían ineludible el recurso a la datación radiocarbónica, enviándose como muestra (HDU-01) un fragmento del ala mayor del esfenoides al Poznan Radiocarbon Laboratory, donde mediante técnica AMS se obtuvo el resultado: Poz-41289: $3290 \pm 35$ BP. La calibración a 2 sigma mediante el programa OxCal v.4.3.2.r.5 (Bronk Ramsey, 2017) y con la curva IntCal13 (Reimer et al., 2013) proporciona el intervalo: $1660(95,4 \%) 1497 \mathrm{cal} \mathrm{BC}$.

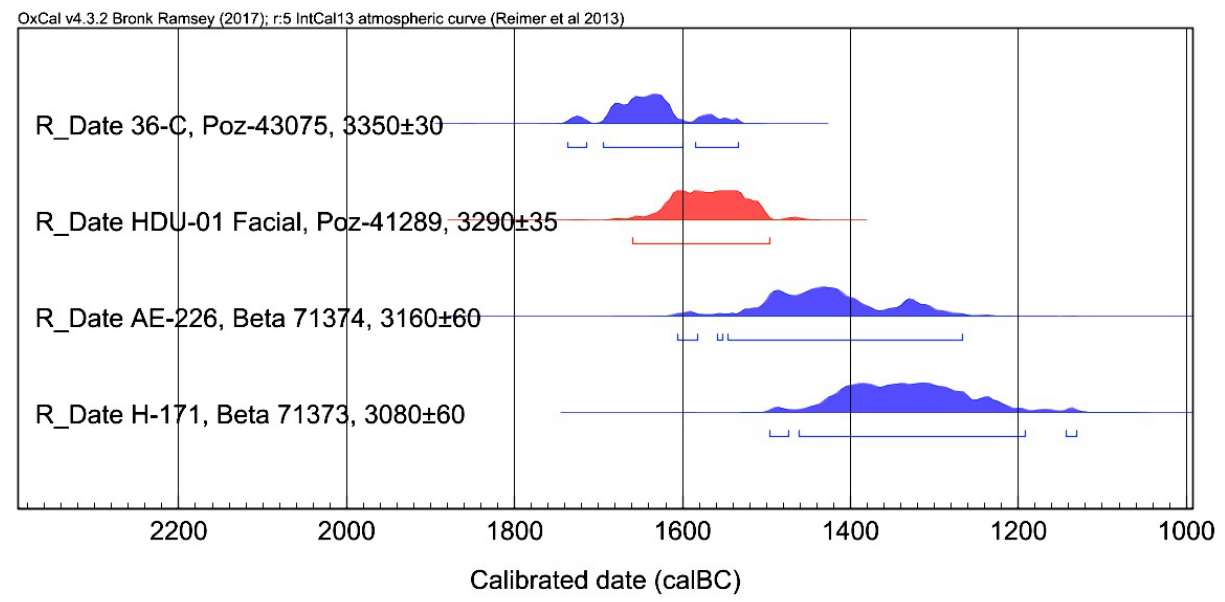

Figura 2. Datación radiocarbónica de la muestra HDU-01 del fragmento fronto-facial de La Huelga y otras tres obtenidas en los hoyos 36-C, AE-226 y H-171 del yacimiento.

exequias), siendo las conductas funerarias solo una parte de las mortuorias. Vid. por ejemplo Zammit, 2003: 187 y 193; Marc, 2016: 55; Schmitt, 2017: 65-6; Pettitt, 2018.
La fecha indica la pertenencia del resto óseo humano al Bronce Medio regional, es decir al Proto-Cogotas I o fase Formativa de Cogotas I, 
y viene a unirse a otras tres obtenidas en el mismo yacimiento de La Huelga, en hoyos en cuyos rellenos había cerámicas cogotenses, el 36-C del sector 2 (Liesau et al., 2014: 95), también de la fase Formativa, y los hoyos H-171 y AE-226 del sector 1 (Pérez Rodríguez et al., 1992: 26), que corresponderían ya a la de Plenitud (Fig. 2).

Corroborada así la pertenencia del resto óseo humano al Proto-Cogotas I, y como paso previo a considerar las posibles interpretaciones del hallazgo, se realiza a continuación su examen bioantropológico.

\section{Consideraciones bioantropológicas}

El fragmento óseo de La Huelga constituye un ejemplo insólito pues, dadas las condicio- nes del hallazgo, nada puede saberse a ciencia cierta de las características del depósito en el que se encontraba, el número mínimo de individuos, si estaba o no acompañado de otros restos humanos y, si fuera ese el caso, el grado de relaciones anatómicas que habría tenido con ellos; pero también conviene recordar que las minuciosas inspecciones de las escombreras no depararon el hallazgo de ningún otro hueso. En tales condiciones, y aunque es sumamente aventurado hacer una aproximación al depósito de origen, este ejemplo presenta, sin embargo, una serie de rasgos singulares que, analizados desde una perspectiva bioantropológica (Craig et al., 2005; Gallay, 2012), permiten plantear, con las debidas cautelas, algunas propuestas de trabajo.
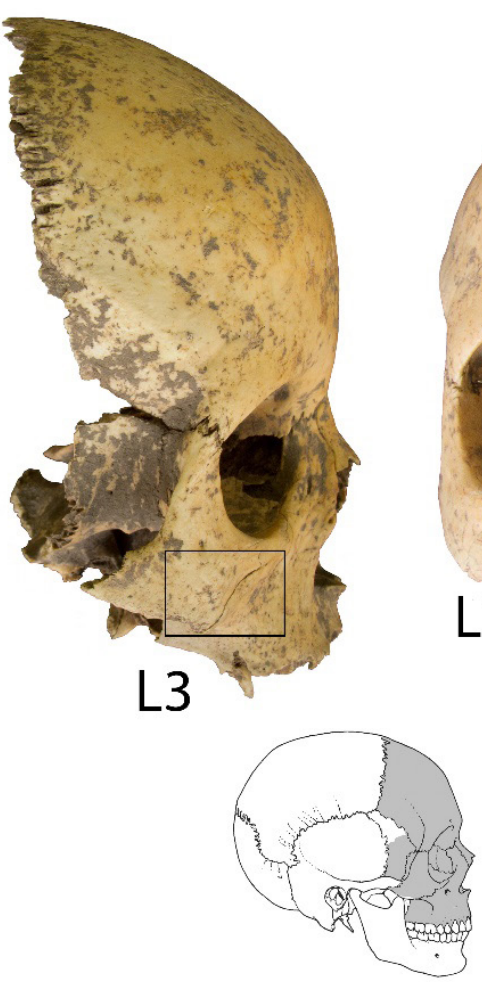
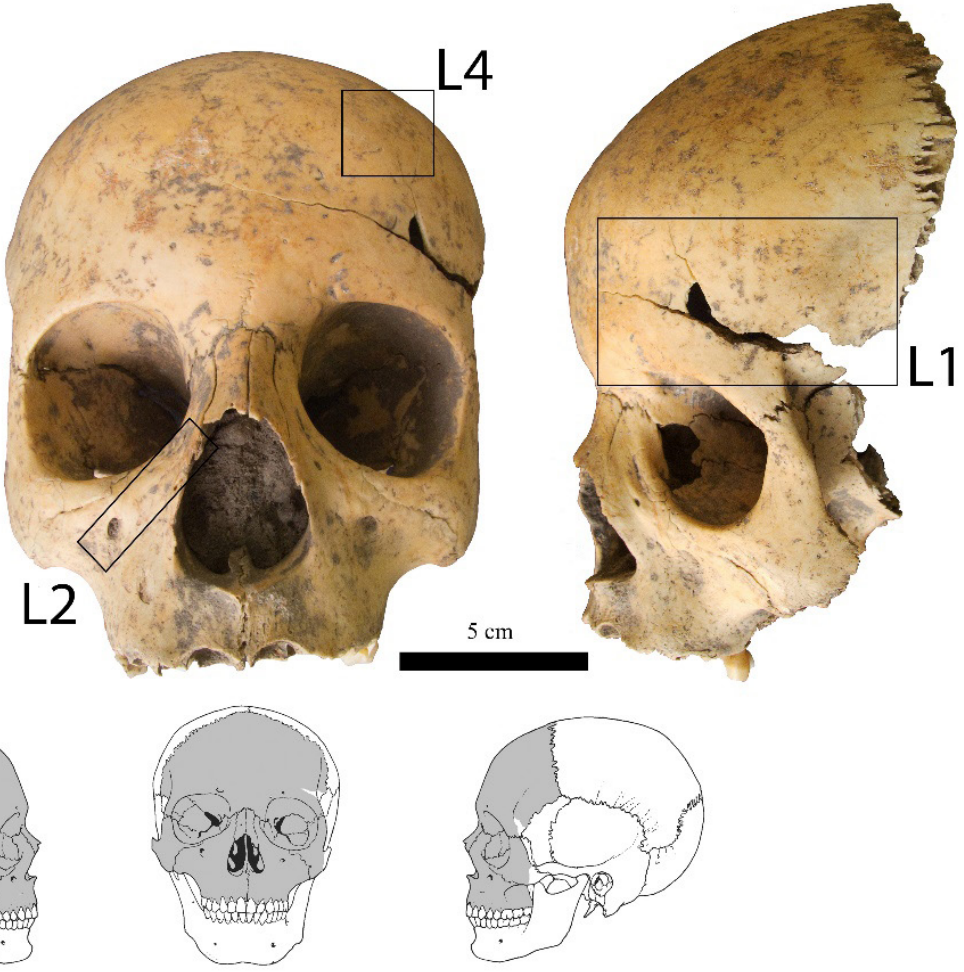

Figura 3. El fragmento craneal de La Huelga en vistas frontal y laterales. Abajo, esquema de representación anatómica.

\subsection{Caracterización y tafonomía del fragmento craneal}

El material humano recuperado correspondería, a juzgar por las piezas dentales presentes en el maxilar, a un individuo fallecido aproximadamente entre los 7-8 años, siendo imposible determinar el sexo ${ }^{5}$. Como se observa en

Una muestra de diente de este individuo fue analizada en su tesis doctoral por Sara Palomo Díez (2015). No hubo resultado en el esquema de la Fig. 3, el grado de representación anatómica es muy parcial, limitándose

cuanto a sexo en la prueba de la amelogenina, pero sí se pudo determinar su haplotipo mitocondrial (16189C 263G 315.1C), correspondiente al haplogrupo $\mathrm{H} 1$, uno más de los reconocidos hasta ahora en los análisis arqueogenéticos de esqueletos de Cogotas I. Presente en la Península ya desde el Calcolítico (Szécsény-Nagy et al., 2017), recientemente se ha detectado el mismo haplogrupo mitocondrial H1 en un individuo de Tordillos (Aldeaseca de la Frontera, Salamanca), también del Cogotas I Formativo (Olalde et al. 2019, muestra I3493/S-EVA26050). 
a buena parte del esplacnocráneo (frontal, zigomático, nasal, maxilar, parte del esfenoides derecho...), con pérdida post mortem de la mayoría de las piezas dentales.

Desde el punto de vista tafonómico, presenta unas buenas condiciones de conservación, apreciándose en superficie la acción de raíces, así como carbonataciones y adherencias de sedimentos, tanto en la región endocraneana como en el exterior. No presenta signos de rodamiento, salvo algunas huellas puntuales que pueden deberse al contacto con el sedimento e, incluso, erosiones puntuales muy localizadas del tejido óseo. Por otro lado, y de especial interés para la discusión de los depósitos de Cogotas I, no hay signos de exposición subaérea, como tampoco de alteración térmica.

Atendiendo al material recuperado, en su lado derecho conserva maxilar, zigomático y parte del ala mayor del esfenoides, ligeramente fragmentado, en los que se han conservado prácticamente todas las suturas (Fig. 3). El frontal se presenta completo, salvo una ligera porción fragmentada en el lado izquierdo, manteniendo la mayor parte de la sutura coronal. En el lado izquierdo se preserva solo la mitad anterior del ala mayor del esfenoides, mientras que el zigomático se encuentra fracturado en su zona posterior, con la consiguiente pérdida del área de sutura. Este lado del maxilar también está ligeramente fragmentado.

Aceptando que era el único resto humano presente en un supuesto hoyo de origen podría pensarse que se trataba de uno más de los depósitos secundarios de cráneo o porción craneal conocidos en el ámbito de Cogotas I. Pero este ejemplo de La Huelga cuenta con otros elementos que lo singularizan, pues el hueso presenta una serie de huellas de origen antrópico que, por su naturaleza, pueden calificarse de peri mortem y cuya explicación apuntaría, a priori, a diferentes agentes causales. En este caso, peri mortem indicaría que se trata de unas alteraciones que se produjeron cuando los tejidos óseos afectados todavía presentaban unas condiciones semejantes a las que tendría en vida del individuo o al menos claramente distinguibles de las que son características al hueso seco (Sorg, 2019).

\subsection{Huellas antrópicas sobre el fragmento craneal}

Existe una amplia literatura (Galloway et al., 2014; Kranioti, 2015; Moraitis et al., 2008;
Spencer, 2012; Wheatley, 2008) dedicada a la distinción de las facturas peri mortem de aquellas con origen tafónomico o las que se produjeron ante mortem. El primer elemento que las caracterizaría es la ausencia de cualquier signo de cicatrización, si bien por lo general se consideran fracturas peri mortem cuando se identificaban algunos -preferentemente dos o más- de los siguientes factores diagnósticos: adherencia o permanencia de pequeños fragmentos óseos en la lesión, ángulos oblicuos (agudos u obtusos) de los márgenes de fractura, delaminación o biselado cortical, textura lisa o bordes de fractura biselados, contorno de fractura regular, fracturas radiantes y concéntricas desde el punto de impacto y superficie de la fractura con la misma coloración y pátina que el hueso circundante.

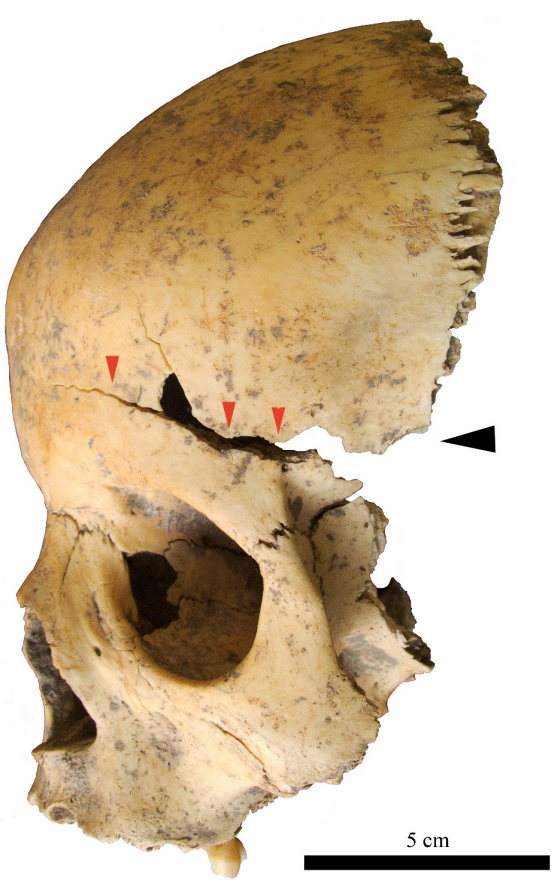

Figura 4. Vista lateral izquierda de la Lesión 1. La flecha negra indica punto de impacto y la pérdida de hueso asociado. Las rojas indican la línea de fractura generada por el trauma peri mortem.

Atendiendo a las lesiones peri mortem de este ejemplar de La Huelga, destaca la presencia de distintas líneas de fractura que surcan el frontal en diferentes puntos (Fig. 4), desde la sutura coronaria en dirección anterior y que afectan tanto a la tabla interna como a la externa (Wedel y Galloway, 2013), que hemos denominado Lesión 1 (L1). La ausencia de los parietales y del temporal iz- 
quierdo impide localizar el punto exacto en el que se iniciarían estas fracturas, pues en ningún caso se habrían originado en el frontal. A juzgar por sus características parecen ser el resultado de impactos controlados que se localizarían fuera del hueso conservado irradiando desde allí a la zona anterior del cráneo, como se describe en distintos ejemplos arqueológicos (por ejemplo, Bello et al., 2016; Marginedas et al., 2020; Santana et al., 2019). Impactos para los que puede reconocerse una energía destacada pues las líneas de fractura superan la sutura coronaria (Wedel y Galloway, 2013), disminuyendo esa energía a medida que avanzan por el frontal. Se contabilizan al menos cinco de estas lesiones, siendo la más importante la que se localiza en el lateral izquierdo (Fig. 4), coincidiendo con el único punto en el que, a juzgar por el aspecto del hueso, se ha conservado parcialmente el punto de impacto, resultado de un trauma contuso que provocó el daño descrito (Fernandez-Jalvo y Andrews, 2016; Bello et al., 2011).

Tabla 1. Criterios para determinar el carácter peri mortem de las lesiones observadas

\begin{tabular}{|c|c|c|c|c|c|c|c|c|}
\hline & Adherencias & $\begin{array}{c}\text { A. oblicuo/ } \\
\text { biselado }\end{array}$ & Textura lisa & $\begin{array}{c}\text { Fractura } \\
\text { regular }\end{array}$ & $\begin{array}{c}\text { Frac. radial/ } \\
\text { concéntrica }\end{array}$ & Color & Delaminación & Figuras \\
\hline L1 & Sí & Sí & Sí & Sí & Sí & Sí & Sí & $\mathbf{4} / \mathbf{5}$ \\
\hline L2 & Sí & - & - & Sí & Sí & Sí & Sí & $\mathbf{6}$ \\
\hline L3 & Sí & - & - & Sí & Sí & Sí & Sí & $\mathbf{7}$ \\
\hline L4 & Sí & Sí & - & Sí & Sí & Sí & Sí & $\mathbf{8}$ \\
\hline
\end{tabular}

Si atendemos a los criterios expuestos previamente para determinar en qué momento se produjeron estas fracturas visibles en el frontal, queda poca duda de que se trata de lesiones peri mortem. (Tabla 1) En ningún caso se pueden asociar a razones tafonómicas o al momento en el que se produjo la alteración reciente del depósito original, pues a las evidencias observables -ángulos agudos y obtusos, retención de porciones de hueso, color semejante, aspecto de los márgenes de la lesión, delaminaciones, etc. (Fig. 5 )- se suma la semejanza de los principales agentes tafonó- micos que afectaron al exterior del hueso y a las zonas expuestas por las fracturadas (Cattaneo y Capella, 2017; Sorg, 2019), como así indicarían el color del tejido óseo o la distribución de carbonataciones en su superficie. En defintiva, todas estas fracturas debieron de tener su origen en impactos directos sobre el cráneo, posiblemente localizados en los parietales, desde los que se generan estas líneas de rotura que terminan en el frontal y que fueron propinados cuando el hueso presentaba buena parte de sus condiciones originales de plasticidad.
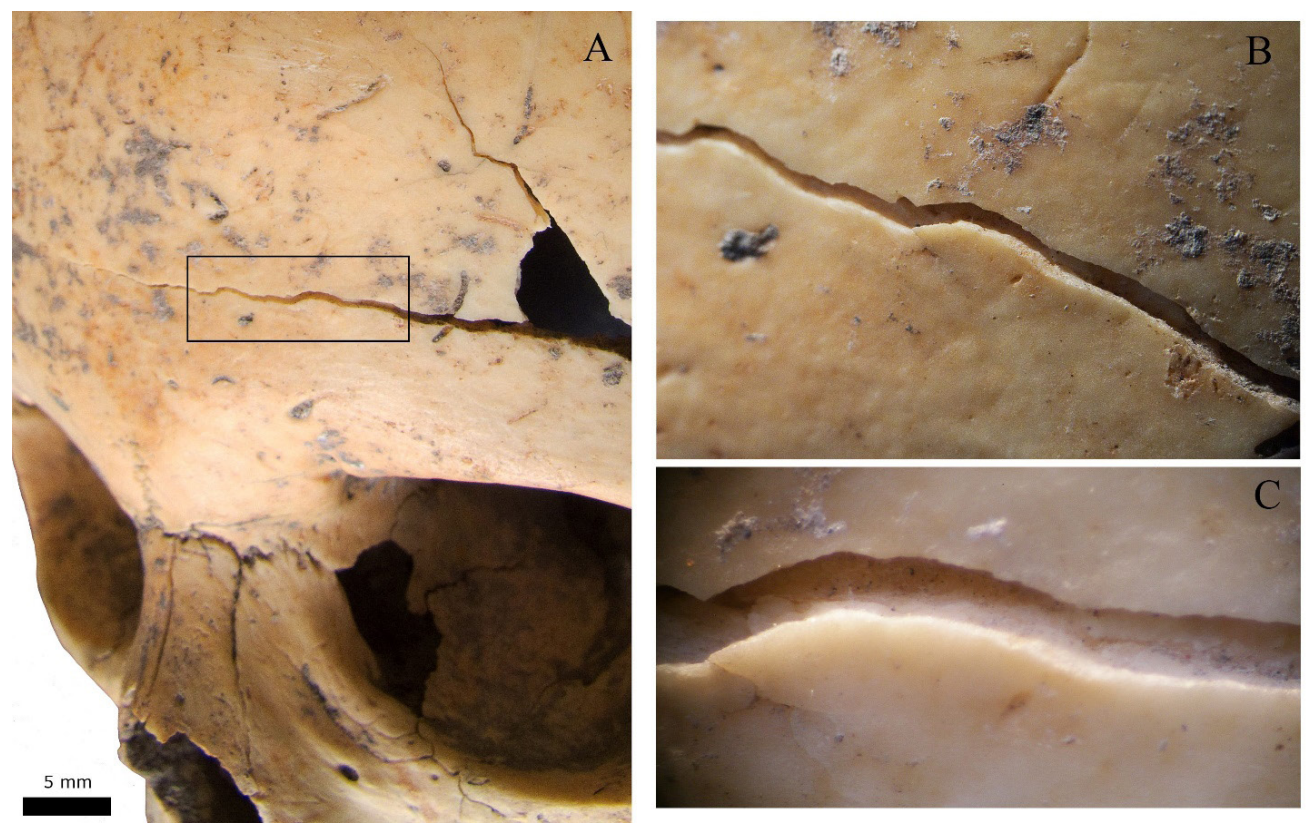

Figura 5. A: Fractura peri mortem en el frontal de la Lesión 1. B: Detalle y C: Vista aumentada (10x) donde se observa el ángulo de la fractura y permanencia de pequeños huesos fracturados. 
El fragmento de La Huelga presenta otras huellas que abundarían sobre lo dicho hasta el momento. En el lado derecho del maxilar se aprecia un impacto que provoca una lesión depresiva en la que el tejido compacto exterior se hunde (Fig. 6 A y C), quedando retenidas pequeñas porciones de hueso que se fracturan por el trauma, la Lesión 2 (Fig. 6). Desde el punto en que recibió el golpe, de aspecto oval, surgen algunas pequeñas fracturas radiales, sobre todo de dirección inferior. Otra lesión semejante se identifica en la zona alta de la apertura nasal (lado derecho), que afecta al maxilar y hueso nasal (Fig. 6 A y B). En el primer caso, con desplazamiento hacia el interior del hueso roto y retención en bisagra de pequeñas partículas óseas, describiendo una línea de fractura que abarca también al nasal (en este caso con la pérdida del hueso fracturado) y que mantiene el mismo color que el tejido óseo circundante.
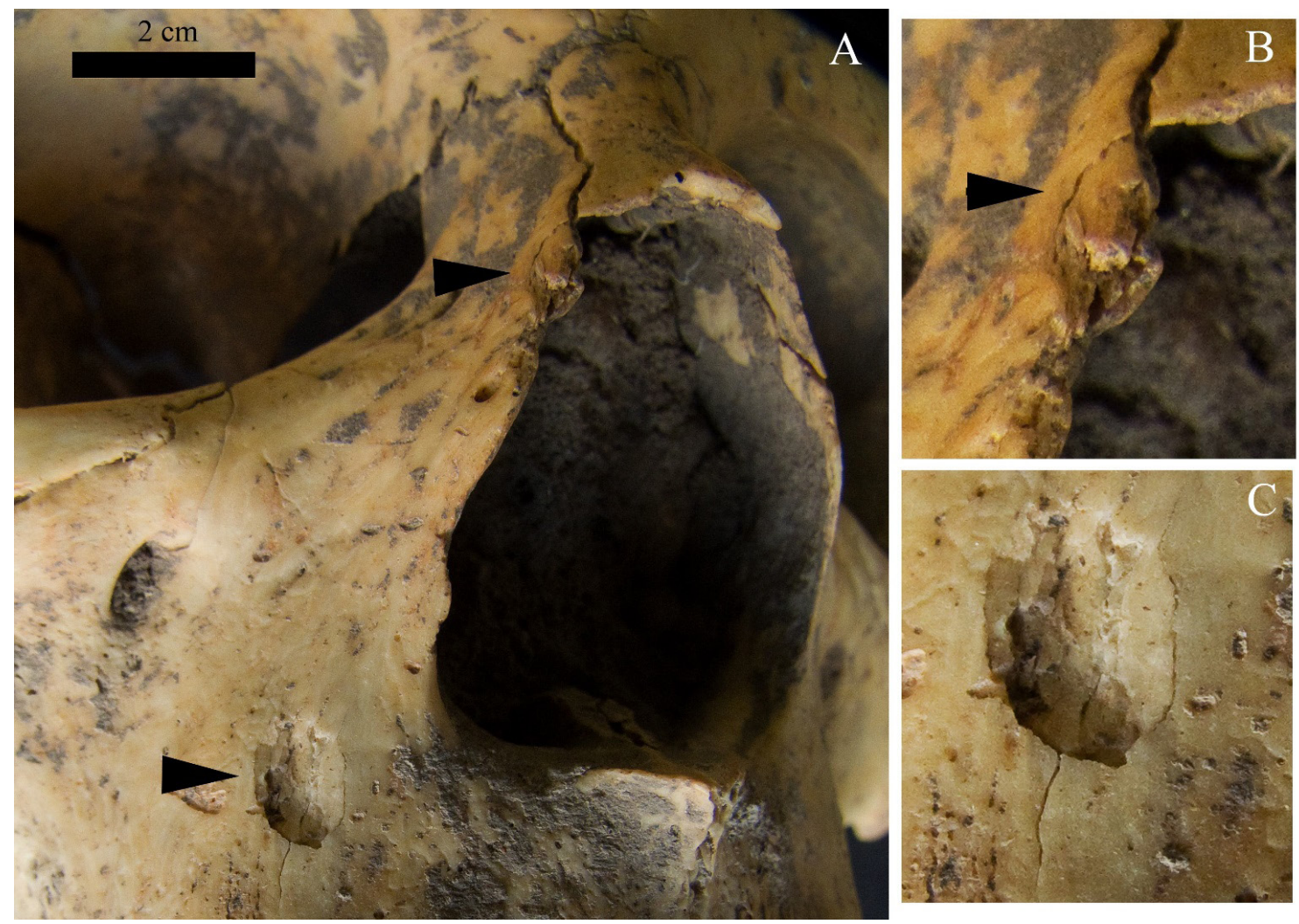

Figura 6. Lesión 2. A: Vista parcial del maxilar derecho con indicación de las fracturas descritas (flechas). B: Detalle de la fractura en la región nasal. C: Detalle de fractura depresiva.

Muy cerca de las anteriores, en la zona de unión del zigomático y el maxilar (Fig. 7), se aprecia un nuevo impacto de un elemento contuso que, como en los anteriores, provoca un hundimiento y la fractura del hueso, la Lesión 3. En este caso, con unas dimensiones algo mayores que los ejemplos previos, se generan fracturas concéntricas y retenciones de pequeñas porciones de hueso rotas por el trauma (Sorg, 2019). Otra huella de impacto, también con retención de parte del tejido afectado, se localiza en el zigomático izquierdo, coincidiendo con la fractura generalizada de esta región y que también puede diagnosticarse como peri mortem.
Finalmente, entre todos los impactos directos observables destaca el localizado en superolateral izquierdo del frontal (Figura 8), la Lesión 4. Se trata de un trauma contuso de aspecto oval de apenas $6 \mathrm{~mm}$ en su eje mayor, que afecta tanto a la tabla interna como a la externa, que aparece ligeramente hundida. Se trata de una lesión de sección troncocónica invertida (Lovel, 1997), con laminaciones del tejido endocraneal y pequeñas fracturas concéntricas al exterior. Se asocia por proximidad a una de las líneas de rotura que recorren el frontal, si bien esta sería posterior a aquella pues parece adaptarse a la lesión en el final de su recorrido. 

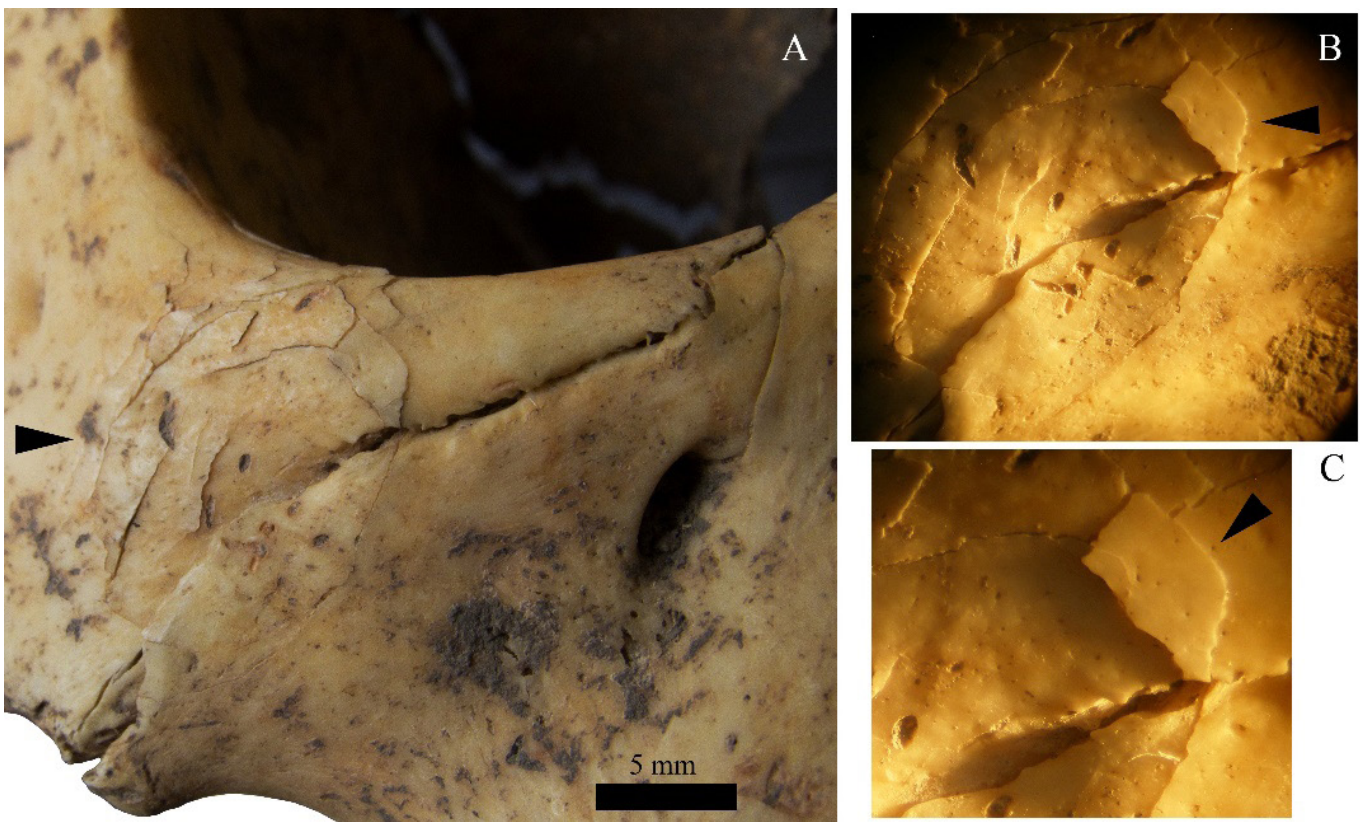

Figura 7. Lesión 3. A: Fractura contusa en la zona de unión entre zigomático y maxilar derecho. B y C: Detalles de la lesión peri mortem (10x).

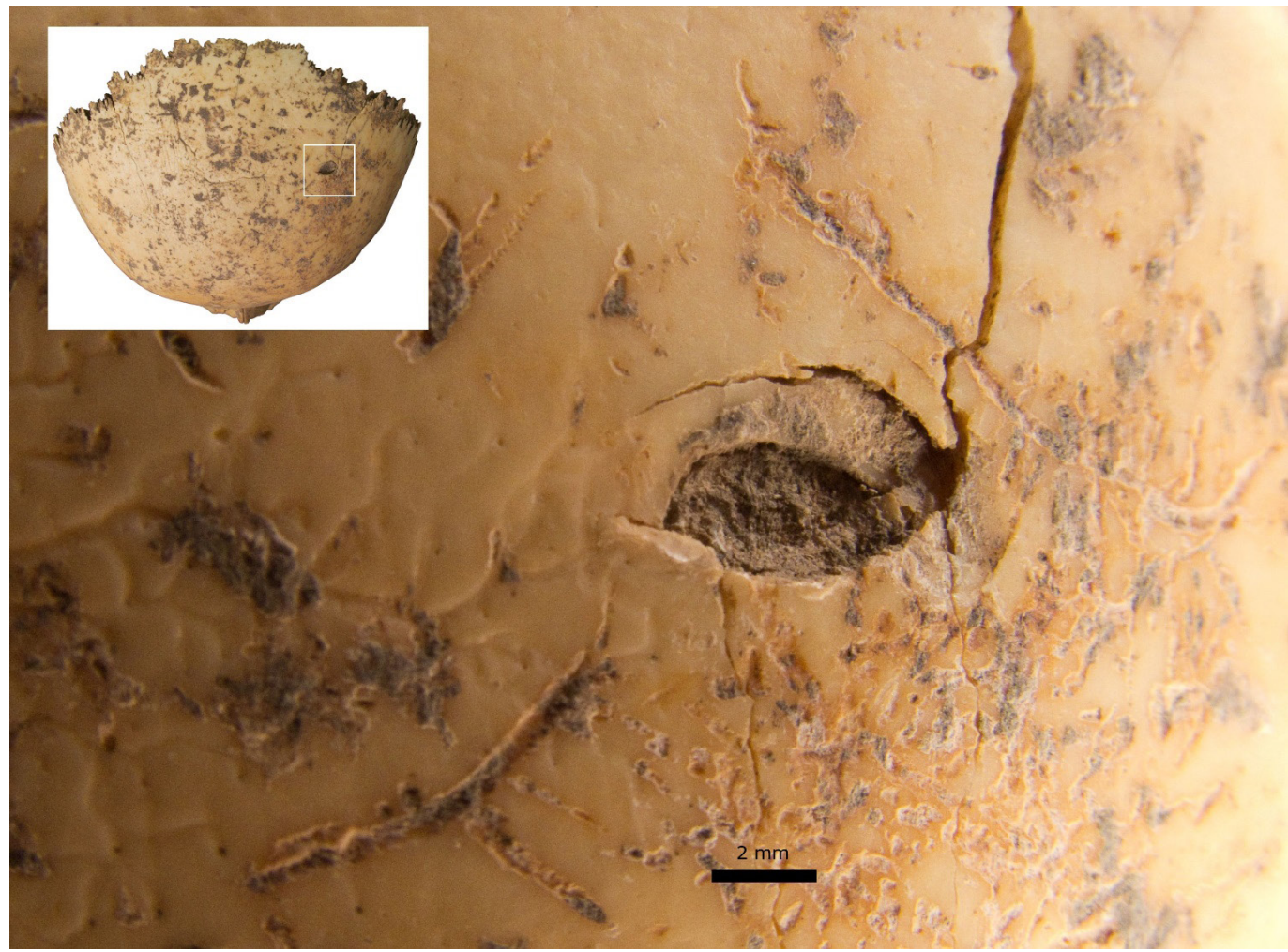

Figura 8. Lesión 4. Detalle de fractura peri mortem en la parte posterior del frontal. Recuadro superior: Vista superior del frontal.

En suma, el fragmento de la Huelga presenta una serie impactos directos sobre el cráneo que afectaron al tejido óseo cuando presentaba unas características semejantes a las que tendría el hueso fresco. Ello no necesariamente significa que se produjeran en el mismo mo- mento de la muerte (o que la provocaran), pues la "frescura del hueso"-retención de humedad y grasas, así como su elasticidad- puede persistir durante días o incluso meses, dependiendo del contexto ambiental en el que se encuentren los restos humanos (Sorg, 2019). 


\section{3 ¿Cómo explicar las huellas antrópicas?}

Son varias las circunstancias que, según el caso, pueden explicar el origen preciso de estos marcadores. De entrada cabría descartar que se trate de una porción casualmente desprendida del resto del cráneo, y más aún que tal cosa se hubiera producido en el momento en el que se alteró el depósito de origen. Ciertamente, por la edad del individuo, la sinostosis de las suturas no se habría producido, y ello habría facilitado la separación del frontal, pero la presencia de fracturas en el lateral izquierdo y otras cercanas a la sutura coronal invitan a pensar en un gesto que, presumiblemente de forma intencional, separó esta región anatómica del resto del esqueleto craneal. Con todo, son varios los escenarios que se presentan como posibles en este caso.

El primero de ellos es que este individuo sufriera traumatismos contusos localizados en distintas regiones del cráneo, que fueron propinados con la intención de provocarle la muerte o le fueron infligidos poco después del óbito. El número de heridas y la dispar localización de los traumas (con preferencia por la región anterior del cráneo) llevarían a pensar que este individuo fue la víctima de un encuentro violento al que no sobrevivió. En otros contextos de Cogotas I ya se han descrito casos de muertes violentas de subadultos (Velasco y Esparza, 2016), lo que haría de este supuesto algo no excepcional. Pero al no disponerse más que del fragmento estudiado y no del resto del esqueleto de este individuo se hace muy complicado validar esta posibilidad, aunque también descartarla por completo; y otro tanto sucede si se plantea la sospecha de que el fragmento de La Huelga fuera el desecho de una práctica antropofágica. En efecto, aunque esa conclusión completamente negativa se ve lastrada por el carácter aislado del fragmento, resultan muy significativos la total ausencia de marcas de corte y un menor grado de fragmentación que el documentado en algunos ejemplos de canibalismo (Boulestin et al., 2009; Cáceres et al., 2007; Bello et al., 2016; Marginedas et al., 2020). Si añadimos también la falta de más restos humanos en la zona en la que se recuperó este ejemplar, todo ello invitaría a calificar de muy poco probables estas primeras suposiciones.

Precisamente atendiendo al número inusitadamente elevado de huellas directas e indirectas de golpes localizados en el cráneo -al menos ocho- puede plantearse que fuera objeto de una intensa manipulación dirigida a obtener la porción conservada separándola del resto del cráneo. La localización periférica de parte de los golpes (en parietales, esfenoides izquierdo, zigomático, etc.) podría ser un primer argumento a favor de este otro escenario. Los situados en maxilar y frontal no parece que respondan a este propósito, salvo que, como luego se discutirá, fuese un subproducto del tratamiento que se dio al cráneo. Si efectivamente esa desconexión hubiera sido el fin perseguido, la ausencia de sinostosis en buena parte de las suturas ayudaría a explicar que los golpes estén espaciados entre sí y que no fuera necesaria una sucesión mucho más intensa para separar esta porción del cráneo (como así sucede, por ejemplo, en los cráneos copa). En este sentido, debemos reseñar que en otros contextos la obtención de piezas de apariencia muy similar a la de La Huelga se conseguía separando o fracturando la sutura coronal y los arcos zigomáticos además de la unión esfenofrontal (Boulestin y Duday, 2012: 149), como así parece suceder en el caso palentino.

Recapitulando lo anterior, el fragmento fronto-facial de La Huelga habría sido intencionalmente separado del resto del cráneo - cuando el hueso estaba todavía fresco- mediante una serie de golpes y fracturas bien localizados, descartándose casi por completo que haya sido el resultado de una conducta violenta o de canibalismo. ¿Cómo interpretarlo entonces?

\section{Discusión}

\section{1. ¿Una sepultura destruida?}

Como se dijo en el apartado 2, esta fue la propuesta de los arqueólogos que excavaron $\mathrm{La}$ Huelga, y sin duda era, en orden a justificar la aparición del resto humano parcial, la forma más lógica en aquel momento de la investigación. Hoy queda descartada, a la vista de las huellas de manipulación detectadas en el examen bioarqueológico Por otra parte, antes de aceptar que pudiera ser el remanente de una sepultura destruida habría que haber despejado algunas dudas, procedentes en primer lugar del carácter totalmente aislado de esa porción ósea, sin ningún otro resto del individuo supuestamente enterrado -lo cual, a la luz del estudio bioantropológico, no parece tafonómi- 
camente justificable-, pero también del recientemente desvelado manejo de algunos huesos selectos para ser utilizados como reliquias acompañantes (Esparza et al., 2018). En esa línea ¿no será el fragmento óseo de La Huelga un elemento único, seleccionado para alguna práctica desconocida?

\section{2. ¿Una 'máscara facial'?}

El fragmento de La Huelga podría ser una 'máscara', denominación descriptiva pero algo equívoca. En efecto, este término se emplea, por ejemplo en la Arqueología Americana, en un sentido muy preciso -cráneos descarnados con los orbitales y la apertura nasal ocupados por objetos remedando los ojos y la nariz-, del que debemos alejarnos. En cambio, podría emplearse ese término en el sentido habitual para ciertas manifestaciones frecuentes en el ámbito de La Tène como son las 'máscaras faciales', es decir, porciones seleccionadas del cráneo consistentes esencialmente en el esplacnocráneo y el frontal, desconectados o fracturados de los huesos adyacentes (vid. Boulestin y Duday, 2012: 149; Rousseau, 2012: 123). Desde luego, aunque en el campo de los estudios celtas el término 'máscara', e incluso 'máscara ritual', está bibliográficamente consolidado, se desconoce a ciencia cierta cual fue su función, sospechándose que pudieron haber estado colocadas o suspendidas, acompañadas por objetos singulares, etc., en santuarios, como es el caso de los de Châtelliers du Vieil-Auzay (Large y Boulestin, 2016) o de Corent (Rousseau, 2012: 123).

En diversos contextos más antiguos y menos complejos, no faltan tampoco las menciones de porciones craneofaciales, por ejemplo, en el Neolítico del norte de Francia, en el yacimiento Chassense de Jonquieres (Poulain y Lange, 1984: fig. 1), o en el de Rumania, en el yacimiento de Cârcea, de la cultura de StarčevoKörös-Criş (Ion et al., 2009: figs. 20-22). Hay algunas referencias también para etapas más recientes, como las que recoge en su recopilación Camps-Fabrer (1993:106), de dos porciones faciales de adultos de Madja-Hraskova (Eslovaquia), ya de época hallstáttica o algo anteriores (Boulestin y Duday, 2012: 149).

Por fortuna, algunas veces se ha ido más allá de la datación y el análisis osteológico. Así, acerca de "a mask made from the skull of a woman" (Burlacu-Timofte y Gogaltan, 2016: lam. III.2) hallada en una estructura del poblado fortificado de Veselé-Hradisko-Podzámske, de la cultura de
Mad'arovce del Bronce de Eslovaquia, los investigadores reclamaban la necesaria cautela -que tenemos bien presente- para interpretar este tipo de restos como parte de la basura corriente, dada la dificultad de deslindar, sin buena información, entre los restos de rituales funerarios irregulares, de rituales no funerarios, de otros procesos de descarte e incuso resultantes de procesos no antrópicos (ibid.: 107-8). Y en otra ocasión, en el yacimiento irlandés de King Stables, del Bronce Final, se menciona una máscara, sobre la que luego se volverá, recortada de un cráneo juvenil y depositada en una "piscina ritual" (Lynn, 1977). No faltan tampoco otros hallazgos interpretados en clave ritual en Escocia, como un fragmento de frontal hallado en Sculptor's Cave, en niveles del Bronce Final, para el que se apunta que pudo haberse exhibido con otros restos de subadultos a la entrada de la cueva (Armit et al., 2011: 271-2 y fig. 16); o como el frontal de adulto de la especial estructura 3 de Cnip Headland, en las Hébridas, datado por radiocarbono $(3215 \pm 30 \mathrm{BP})$ en el Bronce Medio, pero redepositado en un contexto doméstico del Hierro, hacia el cambio de Era, circunstancia en la que Armit y Shapland (2015: 41-43, fig. 8 ) ven el encuentro de una comunidad de la Edad del Hierro afrontando su propio pasado.

En la Península Ibérica estas máscaras no parecen ser abundantes. Es verdad que la bibliografía muchas veces adolece de imprecisión en cuanto a los pormenores de antropología física -por ejemplo, se alude a la aparición de "un cráneo aislado", sin más detalles, en el yacimiento campaniforme de El Juncal, en Getafe (Martínez Calvo et al., 2015: 251)-, por lo que las citas expresas de esplacnocráneos resultan tan sugerentes como escasas y poco detalladas: como lejano antecedente pueden citarse un hallazgo correspondiente al Paleolítico Superior, la porción craneana perteneciente a la cara de un individuo infantil magdaleniense de la cueva de El Pendo (Blas Cortina, 1997: 158); o, ya en el Neolítico Antiguo, el frontal de un individuo infantil también mencionado como "máscara", hallado junto a un feto de ovino en la Cova de Els Trocs, en el Pirineo oscense, (Rojo Guerra et al. 2013: 17 y 46). Más interesante por su mayor cercanía en el espacio y el tiempo podría citarse el individuo $\mathrm{n}^{\circ}$ 1 del enterramiento E1-01-I del área 31 del yacimiento campaniforme de Camino de las Yeseras (Madrid), un enterramiento de tipo hipogeo que habría sido expoliado por las propias gentes campaniformes (Gómez Pérez et al., 2011: 113). Los restos de este individuo -que había sufrido 
en vida una fractura que le ocasionó una desviación de la nariz (ibid.: fig. 44)- consisten esencialmente en un esplacnocráneo con frontal, pero este caso no es totalmente seguro, ya que es el resultado de una laboriosa reconstrucción a partir de numerosos fragmentos y esquirlas (vid. también Liesau y Blasco, 2019: 190 y fig. 5).

Para terminar, también podría traerse a colación un fragmento con parte de la cara, el P22-158, de un infantil de 8 años hallado en la cueva burgalesa de El Mirador, en Ibeas de Juarros, en un contexto del Bronce Antiguo, que ha sido relacionado, como luego veremos, con prácticas de antropofagia (Cáceres et al., 2007: 938 y fig. 7a), ajenas a nuestro caso.

Como ha podido advertirse, las supuestas máscaras carecen de pruebas acerca de su utilización, a no ser las que han sido halladas en contextos con vestigios de ritos, donde puedieran haber sido emplazadas, colgadas, colocadas con ofrendas, etc. A falta de indicios de esa índole para el fragmento de la Huelga, puede plantearse todavía otra hipótesis

\section{3. ¿Un subproducto abandonado?}

El hallazgo de La Huelga es especialmente problemático, y tal vez engañoso, porque su carácter aislado casi obligaba a centrar la atención en el propio resto óseo, y a dotarle de un valor per se, por ejemplo como 'máscara facial' que supuestamente hubiera tenido un papel importante en alguna práctica social.

Pero un enfoque más amplio nos llevaría por otros derroteros, al considerar la excepcionalidad de este hallazgo frente a la repetición de otros algo diferentes, como son los cráneos -especialmente, las calvarias y calotas, y fragmentos neurocraneales-que han venido apareciendo en diversos yacimientos de Cogotas I (Fig. 9), como el de Pico Castro, sin ir más lejos. En efecto, excavando en este prominente yacimiento situado en la misma localidad de Dueñas, concretamente en el hoyo S-38, en cuyo relleno había cerámica con decoración de boquique y línea cosida, apareció una calota de un individuo juvenil masculino (Crespo et al., 2005: 34 y lám. 3c); y en el hoyo S-39 (ibid.: 37), también con cerámicas de boquique y excisas, se halló un neurocráneo infantil muy fragmentado así como una tibia de adulto, que parece una reliquia por su datación radicarbónica mucho más antigua que la del subadulto (Esparza et al., 2018: 358-9). Sin salir de la cuenca del Duero, en el hoyo 75 del Soto de Tovilla (Tudela de
Duero) fue encontrado un cráneo aislado de un adulto joven (Cruz Sánchez et al., 2008), para el que hemos obtenido la datación radiométrica que lo sitúa en el Proto-Cogotas I; en la ciudad de Zamora, la bóveda craneana de un individuo femenino adulto acompañaba a un esqueleto infantil en un hoyo de la plaza de la Catedral con materiales de la Plenitud Cogotas I (Alonso y Centeno, 2005; Caro Dobón y Fernández Suárez, 2007; Esparza et al., 2018: 355-6); y recientemente han sido halladas en Carricastro (Tordesillas) dos calotas craneanas, en sendos hoyos con materiales del Proto-Cogotas I (Patrimonio Inteligente, 2019: ficha u.e.PL3/Hoyo14) y de la Plenitud (ibid.: 141 y lám 6.7.5). En la Submeseta Sur sucede otro tanto en La Dehesa (Alcalá de Henares), también 'campo de hoyos' de Cogotas I, en cuyo hoyo 3 se hallaron dos cráneos, sin mandíbula ni vértebras cervicales, en disposiciones contrapuestas y acompañados de un vaso cerámico y un molino lítico (Macarro, 2002: lám. 19; Marinas, 2014: 44-45 y 132); y también en Yuncos (Toledo), donde se conoce el cráneo sin mandíbula del individuo 2, infantil, hallado en el Fondo 3 del Sector 22, en cuyo relleno hay también cerámicas del ProtoCogotas I (Barroso et al., 2014: 120 y figs. 3 y 4). La lista podría seguramente engrosarse con otros tres hallazgos, pues se han documentado otros fragmentos de neurocráneos, seguramente pequeños, en el hoyo 9 de El Negralejo (RivasVaciamadrid), con cerámicas de Cogotas I Pleno (Blasco Bosqued, 1983: 108) y en los hoyos 112 (Cogotas I Pleno) y 56-57 (Cogotas I Formativo) de la Fábrica de Ladrillos, en Getafe (Blasco Bosqued et al., 2007: 64). En definitiva, el balance entre hallazgos de porciones neurocraneales y de esplacnocráneos en yacimientos de Cogotas I parece en la actualidad claramente desequilibrado, ocho a uno, e incluso doce a uno si se cuentan los fragmentarios ${ }^{6}$.

\footnotetext{
A la lista podría haberse agregado otro caso, el del enterramiento de Renedo de Esgueva (Wattenberg 1957; Palol y Wattenberg 1974: 27-28). En efecto, en las noticias de prensa donde se relata el hallazgo-Libertad (Valladolid), 27 de marzo de 1957, p. 7; Imperio (Zamora) 28 de marzo de 1957, p. 8; $A B C$ (Madrid), 30 de marzo de 1957, p. 35- se insiste en que el esqueleto tenia una especial configuración del cráneo, pues "carecía de frontal". Este interesante detalle, que daría pie a suponer una práctica cultural que comenzaba con la extracción de esa parte del neurocráneo, no puede verificarse, pues de los huesos encontrados unicamente la mandíbula pasó al Museo Arqueológico de Valladolid.
} 


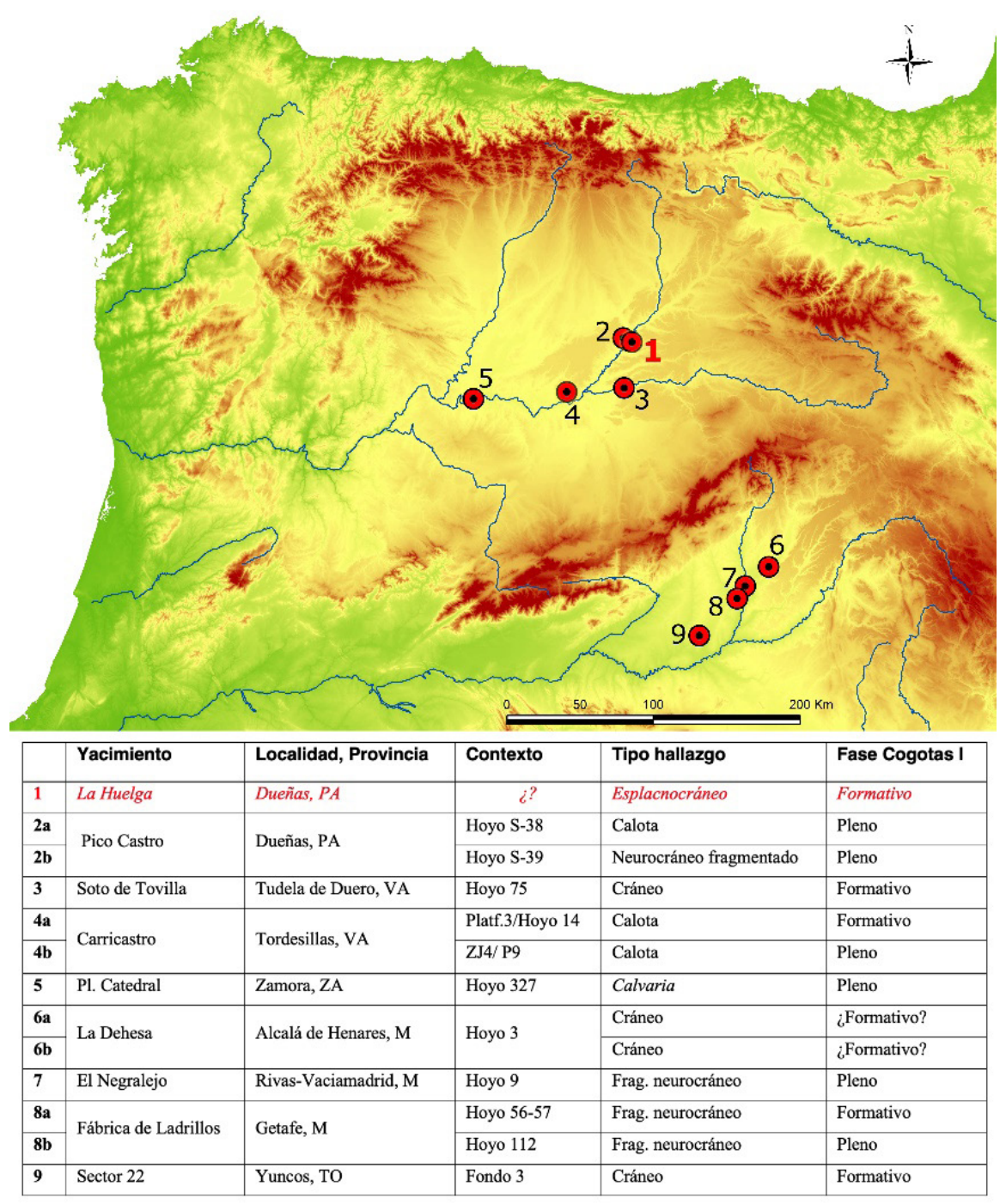

Figura 9. Hallazgos de restos humanos craneales en yacimientos de Cogotas I: mapa y resumen de características.

En este repaso no puede soslayarse el hecho de que en la sierra de Atapuerca -no sin controversia en cuanto a su pertenencia a la 'Zona de Contacto' de Cogotas I o a comunidades vecinas- se han documentado restos humanos en dos cavidades, la Cueva de la Revilla (Abarquero et al., 2005; Moral del Hoyo et al., 2003-4: 70-71) y la Cueva del Mirador (Verges et al., 2002; Moral del Hoyo et al., 20034). En esta última, durante la fase MIR-5, del Bronce Antiguo, fue procesado un importante conjunto óseo con una fuerte representación de elementos craneales -tres docenas-, algunos completos y varias calotas, habiendo solo un fragmento con parte de la cara, el P22-158, de un infantil de 8 años, citado sobre estas líneas. Estos huesos humanos, presentaban, al igual que los de fauna, características marcas de cor- te, fracturas, mordeduras e incluso de huellas de cocinado, que se ajustarían a una conducta antropófaga, de "canibalismo gastronómico" (Cáceres et al., 2007), -interpretación recientemente cuestionada al no poderse descartar su correspondencia a prácticas simbólicas (Saladié y Rodríguez-Hidalgo, 2017; Marginedas et al., 2020)-, siendo los restos objeto de enterramiento secundario posteriormente, ya en el Bronce Medio. Cerca de allí, en la cueva del barrio de La Revilla, en el enterramiento colectivo se señala la presencia de cráneos, neurocráneos, alguna calota, etc. Aunque el carácter del osario no permite despejar rotundamente su carácter intencional (Velasco y Delgado, 2005), sí parece haber algunos elementos anatómicamente desconectados, como tres cráneos sin mandíbula y un fragmento de 
frontal infantil agrupados en un "nido", así como una calota y otros dos cráneos asociados (Abarquero et al., 2005: 101-102).

Puede defenderserse, en definitiva, que el interés por el esqueleto de la cabeza, especialmente cráneos y calvarias, debió de gozar de un notable arraigo en esta zona en momentos anteriores o sincrónicos del Proto-Cogotas I, constituyendo un posible antecedente para los hallazgos inequívocamente Cogotas I.

Así las cosas, la impresión que, para concluir, podría desprenderse es que el verdadero objeto de deseo pudo ser el neurocráneo, y que el esplacnocráneo, que en La Huelga nos ha llegado de forma excepcional, era simplemente un subproducto, el resto sobrante de las operaciones -desensamblaje, fractura, recorte, etc.conducentes a la preparación de calotas o calvarias. A favor de esta posibilidad podríamos argumentar la presencia de impactos directos en el esqueleto facial de La Huelga (frontal, maxilar, etc.) que quizás sean el resultado de los gestos dirigidos a su separación del resto del cráneo. Si el fragmento fronto-facial hubiera sido el objetivo último de la manipulación, los impactos probablemente no se habrían localizado en él, sino en los huesos colindantes en el resto del cráneo.

\section{4 ¿Prácticas sociales de fragmentación y despersonalización?}

Quizás chocaría a un observador actual el que se despreciase esa zona tan concreta del esqueleto craneal, la que corresponde a la cara y la frente, que muy probablemente consideraríamos lo más distintivo de un individuo viviente. Pero frente a esa simplista impresión, conviene recordar a Madgwick (2008) quien, apoyándose en trabajos etnográficos, propuso en su análisis de los huesos humanos en la Edad del Hierro de Wessex que la fragmentación del cuerpo estaría indicando una práctica social deliberada: "Ceremonies involving whole communities, surrounding the symbolic depersonalisation of the individual would serve to further reaffirm the tightly bounded nature of society" (ibid.: 110). Así pues, los restos secundarios no serían objetos desechados, ni despreciables, sino seleccionados para desempeñar un papel importante en ceremonias de índole comunitaria. Entonces, volviendo a la supresión de la parte del rostro en el caso de $\mathrm{La}$ Huelga y los otros yacimientos de Cogotas I, ¿no se podría entender que lo que se pretendía era precisamente eliminar lo individual, enfatizando el carácter comunitario de los depósitos de calotas u otros restos neurocraneales?

Tal es la interpretación propuesta por Cleary (2014: 49) para la conducta de de-facing observada en el sitio irlandés de Ballinderry, un yacimiento del Bronce Final en el cual, en relación con unas estructuras de madera, se hallaron tres cráneos intencionalmente desprovistos del esplacnocráneo (Howells, 1942).

La autora irlandesa recoge también (Cleary, 2014: 51-3) el ya citado caso de King Stables asimismo del Bronce Final (Lynn, 1977), de especial interés porque consiste precisamente en el esplacnocráneo de un joven varón adulto, cortado para separarlo del resto (Delaney, 1977) y que, erosionado, terminaría depositado en una piscina de caracter ritual junto a ofrendas faunísticas, siendo éste el contrapunto de los otros hallazgos craneales, desprovistos de cara, hallados siempre en contextos "seculares" (Cleary, 2014: 53).

Pero, además de las mencionadas cabrían también -al menos en teoría- otras posibilidades de interpretar la contraposición en el tratamiento de porciones craneales, como la que se ha señalado en un trabajo sobre el yacimiento de Tell Qarassa, perteneciente al PPNB siriopalestino (Santana et al., 2012). En este contexto, caracterizado por la enorme importancia concedida al rostro humano, tanto en esculturas como en 'máscaras faciales' y cráneos con la cara modelada en barro, se ha observado en el citado yacimiento la supresión deliberada del viscerocráneo, con el énfasis puesto en cambio en fragmentos neurocraneales. El contraste se ha interpretado (ibid.: 213) como un indicio de tensión social. En efecto, si aquellas representaciones de la cara habrían simbolizado la presencia real de los difuntos posibilitando además la interacción entre los vivos y los muertos, la mutilación del esqueleto facial vendría a impedir esa interacción. Tal comportamiento, observado en el depósito de Tell Qarassa pero también en esculturas de ciertos yacimientos, entre ellos el de Göbekli Tepe, es entendido en términos de "hostilidad ritual", como una forma de venganza o también de protección de los vivos frente al poder nocivo de los muertos (Santana et al., 2012: 214). Aunque se ha insistido (Santana et al., 2015) en que esa deposición o entierro secundario de restos humanos retirados de las tumbas, frente a las ceremonias de carácter comunal, podrían haber sido efectuados por individuos o grupos 
más pequeños y con propósitos de carácter negativo, otros autores han propuesto que los restos de Göbekli Tepe pudieran corresponder a individuos "marcados", ya fuese en sentido positivo o negativo (Gresky et al., 2017).

En sintonía con las interpretaciones recogidas sobre estas líneas, podría sugerirse -eso sí, sin contar con el mismo grado de conocimiento contextual- que, si se ponía el énfasis en el resto del cráneo, que no nos ha llegado, la extracción del fragmento esplacnocraneal de La Huelga, eliminando así la parte de la cara tan característica de lo humano, pudo corresponder a alguna práctica social: pudiera ser con ese propósito de privación de imaginarias posibilidades, o más bien de ocultación de lo personal o individual, reforzando la primacía de lo comunitario.

\section{Consideraciones finales.}

a) La dificultad que entraña cualquier intento de explicación social de un resto humano "suelto" se acrecienta en este caso por la ausencia de un contexto bien definido. Por ello, conscientes de las limitaciones, hemos planteado este trabajo como un ejercicio de reflexión sobre la compleja y heterogénea casuística de las prácticas sociales que las gentes de Cogotas I debieron de desplegar con la participación de restos humanos; y también como un toque de atención de cara a futuras excavaciones, en las que debería extremarse el rigor en la disección y registro para poder establecer el contexto. Recientemente se ha insistido (Liesau y Blasco, 2019: 185-6) en el efecto que la falta de protocolos de extracción y de flotado o cribado del sedimento suponen en la recuperación de piezas óseas de menor tamaño, fundamentales de cara a la interpretación de conjunto. Con todo, tampoco hay que olvidar que, además del contexto, es imprescindible una perspectiva que combine complejas teorías y métodos, como ilustra el caso del frontal infantil de Els Trocs, anteriormente citado: su asociación a un feto de ovicaprino, llevó a considerarlos como indicio de un comportamiento simbólico relacionado con alguna fórmula funeraria o con canibalismo ritual (Rojo et al., 2015: 184), aunque tras disponer de las dataciones radiocarbónicas de los restos de ambos, se propuso que la "máscara" infantil pudiera responder a una eventual circulación de reliquias a lo largo de varios siglos (Rojo Guerra et al., 2013: 17 y
46). Hoy, el reciente estudio interdisciplinar de los restos humanos de los nueve individuos de la fase I de Els Trocs revela que corresponden a una matanza, siendo ese individuo infantil una más de las víctimas de contundentes golpes (Alt et al., 2020)

b) Si en algún momento ha podido desprenderse la impresión de que el fragmento frontofacial de La Huelga fue un desecho inútil, debe recordarse inmediatamente el tipo de hallazgo, por lo que no hay pruebas para afirmar-ni para negar- que el contexto original fue un vertedero, un midden, etc. Por contra, la presencia en otros yacimientos de restos humanos secundarios en lo que parecen contextos "especiales", depósitos estructurados, etc., permitiría sugerir -también con la misma incertidumbre- la pertenencia del hallazgo de La Huelga a alguno similar, e incluso a cualquier estructura doméstica donde hubiera podido ser guardado en espera de una eventual aplicación en algún festín, práctica mortuoria, etc. Como en otras sociedades prehistóricas, los restos humanos han debido de alcanzar gran valor social, en particular por su trascendencia en la generación de ciertos depósitos (Redfern, 2008) que están lejos de ser simples acumulaciones de desechos (Blanco-González, 2014b: 317-8). Recuérdese en ese sentido que en el propio yacimiento de La Huelga se llevaron a efecto también algunas de esas complejas prácticas, como la que se materializó en el hoyo 36-C, con los restos de dos perros desarticulados y despiezados, y acompañados por algunos restos de vacuno (Liesau et al., 2014).

c) De corroborarse, con nuevos hallazgos, el carácter deliberado de la separación de este tipo de fragmentos de viscerocráneo como el de La Huelga, excluyendo por completo que se trate del mero resultado de la destrucción de alguna sepultura, se habrá avanzado otro paso en la caracterización de las prácticas mortuorias de Cogotas I que, junto a las estrictamente funerarias, integran cada vez más otras modalidades de manipulación de restos, especialmente fragmentarios. En efecto, si la imagen que se va abriendo paso es la de una posible norma funeraria, la exposición de cadáveres, que debió de ser aplicada de forma regular, y un recurso excepcional a los enterramientos en hoyo, reservados para los casos de 'mala muerte' (Esparza et al., 2012b), también otras prácticas relacionadas con la muerte fueron muy importantes para estas poblaciones: en efecto, solían manejar huesos humanos desco- 
nectados, a juzgar por su recurrente presencia en los yacimientos -son ya casi veinte casos en la Submeseta Norte-, y los integraban, en especial los elementos craneales, en relación con eventos constructivos o de abandono de espacios domésticos, con ceremonias de agregación, etc., en definitiva con actos sociales de gran importancia o situaciones particularmente complicadas, como se ha propuesto para otras regiones (Armit y Shapland, 2015). Así, algunos de tales fragmentos parecen haber servido como auténticas reliquias acompañantes de ciertos enterramientos infantiles (Esparza et al., 2018); otros, especialmente los neurocraneales, depuestos en los hoyos anteriormente citados, debieron de usarse en unos casos para ciertas prácticas de ritualización del abandono de una cabaña o sitio - como el fragmento de neurocráneo de Fábrica de Ladrillos que se introdujo junto al mobiliario y restos de revestimientos de una cabaña (Sánchez Polo, 2010: 178-180-) y en ocasiones para ceremonias colectivas de tipo festín (Blanco-González, 2014a: 445).

d) Desde el punto de vista cronológico, estas formas de utilización de restos craneales no parecen ser exclusivas de ninguna fase concreta de las establecidas en Cogotas I. Es verdad que como se ha venido señalando repetidamente (Blasco Bosqued, 1997: 188; Blasco Bosqued y Lucas Pellicer, 2001: 223) si en la Plenitud de Cogotas I se prolongan -aunque enrarecidaslas inhumaciones en hoyo de la fase Formativa, hay una novedad consistente en la esporádica aparición, entre los rellenos de los hoyos, de restos humanos de carácter secundario, despojos humanos muy parciales. Sin embargo, como se observa en la Fig. 9, los parciales, al menos los que ponen el acento en el cráneo, corresponden tanto al Formativo (Proto-Cogotas I) como al Pleno. Salvo una aparente relación de los cráneos completos con el Formativo, no parece tan claro que haya un patrón cronológico, de evolución a lo largo de Cogotas I; antes bien podría tratarse de una diversidad explicable por otras razones, por ejemplo, como variantes de las fórmulas mortuorias a las que se aplicaban los restos humanos secundarios que, más alla de su procedencia concreta -ya sea de expositores, o de la conservación doméstica, de la reapertura de hoyos, etc-, debieron de usarse, como antes se dijo, para fiestas, para ritualizar el abandono, etc.

e) Una reconstrucción precisa de las intenciones de las gentes de Cogotas I resulta impo- sible, en primer lugar por la falta de verdadero contexto, pero sobre todo porque los posibles referentes etnográficos son extraordinariamente variados y hasta contradictorios (Frazer 1934: 5). Ello hace muy difícil el aprovechamiento arqueológico tradicional, como ya señalara Ucko (1969), pero también otros enfoques más rigurosos: por ejemplo, en un reciente estudio de la utilización de cráneos en la cultura Moche, Erica Hill (2006) recogía las más importantes funciones desempeñadas por cabezas o cráneos en el registro etnográfico -apotropaica, sinécdoque, cathexis o talismán, recurso mnemónico, obliteración u olvido, y ofrenda-, cuya pertinencia interpretativa depende obviamente de la disponibilidad de buenos datos bioarqueológicos, iconográficos y contextuales que permitan establecer pautas recurrentes. No siendo este nuestro caso, lo que sí podría entreverse, por el fragmento de La Huelga y otros restos mortuorios de Cogotas I, es que estaríamos, de forma general, ante una concepción del mundo como la que proponen Brück $(2004,2006)$ para el Bronce de las Islas Británicas o Valera (2019) para el Neolítico y Calcolítico del sur de Portugal: a diferencia del mundo moderno, progresivamente regido por una ontología dualista, el mundo prehistórico debió de estructurarse a partir de otras categorías, con gran importancia del principio de participación entre las partes y el todo. En ese sentido, el cuerpo humano sería concebido como algo divisible en múltiples partes, y esa partibilidad conocida en distintas sociedades puede servir para propósitos muy diversos, e incluso opuestos: por ejemplo, para conmemorar o para borrar el recuerdo, para consagrar o para profanar (Geller, 2012: 116), en definitiva, para el reconocimiento o para la reprobación.

f) Para concluir: descartadas o diluidas por su escaso fundamento algunas de las hipótesis planteadas -único resto conservado de una sepultura destruida, 'máscara facial' - resta la posibilidad, en cambio, de que pudiera tratarse del subproducto de la preparación de un elemento neurocraneal (calvaria, calota), y que esa conducta de fragmentación con extirpación de la parte de la cara representase metafóricamente la supresión de lo individual, en el contexto de una práctica performativa de lo comunitario. El caso de La Huelga quedaría así encuadrado en aquellas prácticas mortuorias de Cogotas I que, como se apuntaba hace poco (Esparza et al., 2018: 362), debieron de servir como refuerzo ideológico de la comu- 
nidad en prevención de asomos de jerarquización. Constituiría también un indicio más de la importante continuidad en lo social patente desde el Megalitismo a Cogotas I, apenas interrumpida en el Calcolítico Avanzado: si como sostienen Delibes et al. (2019: 45) las sepulturas del Campaniforme habían puesto "...claro acento en la individualidad -o en una versión distinta de la identidad relacional: la pertenencia a una élite (Hernando, 2002: 160-1)-, minando durante algún tiempo un orden milenario que frenaba la jerarquización (García García, 2017: 215ss)", el hallazgo de La Huelga y los de otros restos craneales ilustrarían el retorno y la vigencia, en la larga duración, de ese mecanismo de control social.

La interpretación que aquí se sugiere para el fragmento de La Huelga - un resto aislado, insistimos- no puede estrictamente ser probada, a no ser que se llegue a encontrar el resto del cráneo, excavando en el propio yacimiento de La Huelga... o en otro, por ejemplo el cercano de Pico Castro, donde algunos hoyos contenían restos neurocraneales de otros individuos. En ese sentido, siendo la identificación de restos del mismo individuo un objetivo actualmente viable con el concurso de métodos osteológicos y genéticos (Haglund y Sorg, 2002: 146), el fragmento de La Huelga consti- tuye un aviso acerca de posibles prácticas mortuorias cuyo reconocimiento es imprescindible para poder desarrollar un marco interpretativo riguroso.

\section{Agradecimientos}

Al Ministerio de Economía e Innovación, por el soporte de los Proyectos del Plan Nacional HAR2009-10105 y HAR2013-43851-P. A la Consejería de Cultura de la Junta de Castilla y León, por los permisos concedidos. A nuestros amigos F. Javier Pérez Rodríguez, F. Javier Abarquero Moras y Carmelo Fernández Ibáñez, respectivamente director, conservador y restaurador del Museo de Palencia, por las facilidades dadas para nuestro trabajo con el fragmento, conservado en el Museo con el número de inventario 1992/2/D/2. Al Prof. Germán Delibes de Castro (Universidad de Valladolid) por su inestimable ayuda. Al Prof. Tomasz Goslar (Poznan Radiocarbon Laboratory) por las dataciones AMS. A Strato S.L. y a Patrimonio Inteligente, por su colaboración; al Prof. Esteban Álvarez Férnandez y a Rodrigo Portero Hernández, de la Universidad de Salamanca, por su apoyo material; y a Verónica Alberto Barroso por el abstract.

\section{Bibliografía}

Abarquero Moras, F. J. (2005): Cogotas I. La difusión de un tipo cerámico durante la Edad del Bronce, Junta de Castilla y León, Valladolid.

Abarquero, F.J.; Palomino, A.L.; Negredo, M.J. (2005): La Cueva de La Revilla, un enterramiento colectivo del Bronce Protocogotas en la Sierra de Atapuerca (Burgos). Boletín del Seminario de Arte y Arqueología, 71(1): 89-136.

Alonso Gregorio, O.; Centeno Cea, I. Ma (2005): Primera intervención arqueológica en el antiguo solar de la Residencia de Nuestra Señora de la Paz, en la plaza de la Catedral c.v. a la Calle Obispo Manso (Zamora). Anuario. Instituto de Estudios Zamoranos Florián de Ocampo, 22: 101-132.

Alt, K.W.; Tejedor Rodríguez, C.; Nicklisch, N. et al. (2020): A massacre of early Neolithic farmers in the high Pyrenees at Els Trocs, Spain. Scientific Reports, 10: 2131 https://doi.org/10.1038/s41598-02058483-9

Armitt, I. (2012): Headhunting and the Body in Iron Age Europe. Cambridge University Press, Cambridge.

Armit, I.; Shapland, F. (2015): Death and Display in the North Atlantic: The Bronze and Iron Age Human Remains from Cnip, Lewis, Outer Hebrides. Journal of the North Atlantic, 9: 35-44. https://doi. org/10.3721/037.002.sp902

Armit, I.; Schulting; R.; Knüsel, C.J.; Shepherd, I.A.G. (2011): Death, Decapitation and Display? The Bronze Age and Iron Age Human Remains from the Sculptor's Cave, Covesea, North-east Scotland. Proceedings of the Prehistoric Society, 77: 251-78. https://doi.org/10.1017/S0079497X00000694

Barroso, R.; Bueno, P.; González, A.; Candelas, N.; Rojas, J.M.; López Sáez, J.A. (2014): Enterramientos de la Edad del Bronce en la Meseta Sur peninsular a partir del Sector 22, Yuncos (Toledo). Munibe Antropologia-Arkeologia, 65: 117-136

Bello, S. M.; Parfitt, S. A.; Stringer, C. B. (2011): Earliest Directly-Dated Human Skull-Cups. PLoS ONE 6(2): e17026. https://doi.org/10.1371/journal.pone.0017026. 
Bello, S.M.; Wallduck, R.; Dimitrijević, V.; Živaljević, I.; Stringer, C.B. (2016): Cannibalism versus funerary defleshing and disarticulation after a period of decay: comparisons of bone modifications from four prehistoric sites. American Journal of Physical Anthropology, 161(4): 722-743. https://doi. org/10.1002/ajpa.23079

Blanco-González, A. (2014a): Tracking the social lives of things. Biographical insights into Bronze Age pottery in Spain. Antiquity, 88 (340): 441-455. https://doi.org/10.1017/S0003598X00101103

Blanco-González, A. (2014b): Sitios en altura y vasijas rotas: reconsiderando la etapa de 'plenitud' de Cogotas I (1450-1150 cal AC) en la Meseta. Trabajos de Prehistoria, 71(2): 305-329. http://dx.doi. org/10.3989/tp.2014.12136

Blas Cortina, M.A. de (1997): En torno a los restos esqueléticos humanos del Paleolítico SuperiorEpipaleolítico Cantábrico. Homenaje a Juan Uría Ríu, vol. I., Universidad de Oviedo, Oviedo: 147-170.

Blasco Bosqued, $\mathrm{M}^{\mathrm{a}} \mathrm{C}$. (1983): Un nuevo yacimiento del Bronce madrileño: El Negralejo (RivasVaciamadrid, Madrid). Noticiario Arqueológico Hispánico, 17: 43-190.

Blasco Bosqued, Ma C. (1997): Manifestaciones funerarias de la Edad del Bronce en la Meseta. Saguntum, 30: $173-190$

Blasco Bosqued, M ${ }^{a}$ C.; Lucas Pellicer, M.R. (2001): Problemática del Bronce Final en la Meseta. Spal, 10: 221-233.

Blasco, C.; Blanco, J.F.; Liesau, C.; Carrión, E.; García, J.; Baena, J.; Quero S.; Rodríguez de la Esperanza, Mã. (2007): El Bronce Medio y Final en la región de Madrid. El poblado de la Fábrica de Ladrillos (Getafe, Madrid). Estudios de Prehistoria y Arqueología Madrileñas, 14-15. Museo de los OrígenesSan Isidro, Madrid.

Boulestin, B.; Duday, H. (2012): Acquisition, préparation et autres traitements de la tête chez les Gaulois: aspects anthropobiologiques. Crânes trophées, crânes d'ancêtres et autres pratiques autour de la tête (B. Boulestin; D. Henry-Gambier, dirs.), Archaeopress, Oxford: 139-157. [BAR IntSer 2415].

Boulestin, B.; Zeeb-Lanz, A.; Jeunesse, C.; Haack, F.; Arbogast, R.M.; Denaire, A. (2009): Mass cannibalism in the Linear Pottery Culture at Herxheim (Palatinate, Germany). Antiquity, 83: 968-982. https://doi. org/10.1017/S0003598X00099282

Bronk Ramsey, C. (2017): Methods for Summarizing Radiocarbon Datasets. Radiocarbon, 59(2): 18091833. https://doi.org/10.1017/RDC.2017.108

Brück, J. (1995): A place for the dead: the role of human remains in Late Bronze Age Britain. Proceedings of the Prehistoric Society, 61: 245-277. https://doi.org/10.1017/S0079497X00003091

Brück, J. (2004): Material metaphors: The relational construction of identity in Early Bronze Age burials in Ireland and Britain. Journal of Social Archaeology, 4(3): 307-303. https://doi. org $/ 10.1177 / 1469605304046417$

Brück, J. (2006): Fragmentation, Personhood and the Social Construction of Technology in Middle and Late Bronze. Cambridge Archaeological Journal, 16(3): 297-315. https://doi.org/10.1017/ S0959774306000187.

Burlacu-Timofte, R.; Gogaltan, F. (2016): Human Bone Remains in the Bronze Age tell settlements of the Carpathian Basin. Settlements of Life and Death. Studies from Prehistory to Middle Ages. Editura Mega, Cluj-Napoca: 89-122.

Cáceres, I.; Lozano, M.; Saladié, P. (2007): Evidence for Bronze Age Cannibalism in El Mirador Cave (Sierra de Atapuerca, Burgos, Spain). American Journal of Physical Anthropology, 133: 899-917. https://doi.org/10.1002/ajpa.20610.

Camps-Fabrer, H. (1993): L'emploi d'ossements humains durant l'Holocène sur le pourtour de la Méditerranée occidentale et dans les pays voisins. Prehistoire Anthropologie Mediterranéennnes, 2: 65-117.

Caro Dobón, L.; Fernández Suárez, M.E. (2007): Enterramiento en fosa del Bronce Final en la ciudad de Zamora. Enfermedad, Muerte y Cultura en las Sociedades del Pasado. Actas del VIII Congreso Nacional de Paleopatología (F. J. Barca Durán; J. Jiménez Ávila, eds.), Fundación Academia Europea de Yuste, Cáceres: 91-100.

Cleary, K. (2014): Human bone as material culture of the living. Exploring Prehistoric Identity in Europe: Our Construct or Theirs? (V. Ginn; R. Enlander; R. Crozier, eds.), Oxbow Books, Oxford: 44-59.

Craig, R.C.; Knüsel, C.J.; Carr, G.C. (2005): Fragmentation, mutilation and dismemberment: an interpretation of human remains on Iron Age sites. Warfare, violence and slavery in prehistory (M.P. Pearson; I.J.N. Thorpe, eds.), Archaeopress, Oxford: 165-80. [BAR IntSer 1374], 
Crespo Díez, M.; Herrán Martínez, J.I.; Puente Aparicio, M.J. (2005): Intervención arqueológica en el "Pico Castro", Dueñas, Palencia. $2^{a}$ fase. Octubre-Noviembre de 2005. Trabajos de seguimiento arqueológico. Informe técnico depositado en el Servicio Territorial de Cultura de la Junta de Castilla y León. Palencia.

Cruz Sánchez, P.J.; San Gregorio Hernández, D.; Martín Rodríguez, E.; Martín Vela, R. (2008): Informe correspondiente a los trabajos de seguimiento y excavación arqueológica en el Soto de Tovilla de Tudela de Duero (Valladolid). Campañas 2005-2007. Informe inédito depositado en el Servicio Territorial de Cultura de la Junta de Castilla y León en Valladolid. Valladolid.

Delaney, M. (1977): Report on the human skull fragments, apéndice en Lynn, 59-61.

Delibes de Castro, G.; Esparza Arroyo, A.; Velasco Vázquez, J.; García García, M.; Santa Cruz del Barrio, A.; Misiego Tejeda, J. (2019): Dobles exequias y circulación de reliquias en el Calcolítico Inicial de la Cuenca del Duero: Sobre las inhumaciones parciales del "recinto de fosos" de Santa Cruz III (Cabezón de Pisuerga, Valladolid). Cuadernos de Prehistoria y Arqueología de la Universidad Autónoma de Madrid, 45: 25-48. http://doi.org/10.15366/cupauam2019.45.00

Esparza-Arroyo, A.; Sánchez-Polo, A.; Velasco-Vázquez, J. (2018): Damaged Burials or Reliquiae Cogotenses? On the Accompanying Human Bones in Burial Pits Belonging to the Iberian Bronze Age. Archaeologies: Journal of the World Archaeological Congress, 14(3): 346-376. https://doi.org/10.1007/ s11759-018-9351-0

Esparza Arroyo, Á.; Velasco Vázquez, J.; Delibes de Castro, G. (2012a): HUM 2005-00139: Planteamiento y primeros resultados de un proyecto de investigación sobre la muerte en Cogotas I. Cogotas I. Una cultura de la Edad del Bronce en la Península Ibérica (J.A. Rodríguez Marcos; J. Fernández-Manzano, J., eds.), Universidad de Valladolid, Valladolid: 259-322.

Esparza Arroyo, Á.; Velasco Vázquez, J.; Delibes de Castro, G. (2012b): Exposición de cadáveres en el yacimiento de Tordillos (Aldeaseca de la Frontera, Salamanca). Perspectiva bioarqueológica y posibles implicaciones para el estudio del ritual funerario de Cogotas I. Zephyrus, LXIX: 95-128.

Fernández-Jalvo, Y.; Andrews, P. (2016): Atlas of Taphonomic Identifications: 1001+ Images of Fossil and Recent Mammal Bone Modification. Springer, Dordrecht.

Frazer, J.G. (1934): The Fear of the Dead in Primitive Religion, vol.II. MacMillan, Londres.

Gallay, A. (2012): Pour une problématique des têtes coupées. Crânes trophées, crânes d'ancêtres et autres pratiques autour de la tête (B. Boulestin; D. Henry-Gambier, dirs.), Archaeopress, Oxford: 5-12. [BAR IntSer 2415].

Galloway, A.; Zephro, L.; Wedel, V. (2014): Diagnostic criteria for the determination of timing and fracture mechanism. Broken bones. Anthropological analysis of blunt force trauma (V. Wedel; A. Galloway, eds.), Charles C. Thomas, Springfiled: 47-58.

García García, M. (2017): La Edad de los Metales en el Duero medio: la evolución del paisaje y de las sociedades. Universidad de Valladolid, Valladolid [Studia Archaeologica ${ }^{\circ} 102$ ].

Geller, P. (2012): Parting (with) the dead: body partibility as evidence of commoner ancestor veneration. Ancient Mesoamerica, 23(1): 115-129. https://doi.org/10.1017/S0956536112000089

Georgieva, P.; Russeva, V. (2016): Human Skull Artifacts-Roundels and a Skull Cap Fragment from Kozareva Mogila, a Late Eneolithic Site. Archaeologia Bulgarica, XX, 2: 1-28.

Gómez, J.L.; Blasco, C.; Trancho, G.; Ríos, P.; Grueso, I.; Martínez, Mas. (2011): Los protagonistas. Yacimientos calcoliticos con campaniforme de la región de Madrid: nuevos estudios (C. Blasco, C. Liesau, P. Ríos, eds.), Universidad Autónoma de Madrid, Madrid: 101-132.

Gresky, J.; Haelm, J.; Clare, L. (2017): Modified human crania from Göbekli Tepe provide evidence for a new form of Neolithic skull cult. Science advances, 3(6), e1700564. https://doi.org/10.1126/ sciadv. 1700564

Haglund, W.D.; Sorg, M.H. (eds.) (2002): Advances in Forensic Taphonomy: Method, Theory, and Archaeological Perspectives. CRC Press, Boca Raton.

Hernando Gonzalo, A. (2002): Arqueología de la identidad. Akal, Torrejón de Ardoz.

Hill, E. (2006): Moche Skulls in Cross-Cultural Perspective. Skull Collection, Modification and Decoration (M. Bonogofsky, ed.), Archaeopress, Oxford: 91-100.

Howells, W.W. (1942): Human remains from Ballinderry. Proceedings of the Royal Irish Academy, 47C: 17-20.

Ion, A.; Soficaru, A.; Miriţoiu, N. (2009): Dismembered human remains from the "Neolithic" Cârcea site (Romania). Studii de Preistorie, 6: 47-79. 
Kranioti, E.F. (2015): Forensic investigation of cranial injuries due to blunt force trauma: current best practice. Research and Reports in Forensic Medical Science, 5: 25-37. https://doi.org/10.2147/RRFMS. $\mathrm{S} 70423$

Large, J.M.; Boulestin, B. (2016): Un sanctuaire gaulois aux Châtelliers? En Large, J. M.: Opération Archéologique Châtellers du Vieil-Auzay-Auzay (Vendée). Memoria de excavación, disponible en http:// gvep.fr/wp-content/uploads/2017/04/2016-Rapport-Auzay-Chatelliers-pf.pdf. Acceso el 15/3/2019.

Liesau, C.; Blasco, C. (2019): La manipulación de los ancestros: acerca de los complejos ritos funerarios campaniformes en el centro peninsular. ;Un brindis por el príncipe! El Vaso Campaniforme en el interior de la Península Ibérica (2500-2000 A.C.) (G. Delibes; E. Guerra, eds.): Museo Arqueológico Regional, Madrid, vol. II: 183-204.

Liesau von Lettow-Vorbeck, C.; Esparza Arroyo, A.; Sánchez Polo, A. (2014): ¿Huesos en la basura o depósito ritualizado? Los perros descuartizados de La Huelga (Dueñas, Palencia). Zephyrus, LXXIV: 89-115. http://dx.doi.org/10.14201/zephyrus20147489115

Lovell, N.C. (1997): Trauma analysis in paleopathology. American Journal of Physical Anthropology, 104(S25): 139-170.

Lynn, C.J. (1977): Trial excavations at the King's Stables, Tray Townland, Co. Armagh. Ulster Journal of Archaeology, 40: 42-62.

Macarro, J.A: (2002): La Alcalá prehistórica. El poblado de la Edad del Bronce de "La Dehesa". Fundación Colegio del Rey/Ayuntamiento de Alcalá, Alcala de Henares.

Madgwick, R. (2008): Patterns in the modification of animal and human bones in Iron Age Wessex: revisiting the excarnation debate. Changing Perspectives on the First Millennium BC: Proceedings of the Iron Age Research Student Seminar 2006 (O. Davis; N. Sharples; K.E. Waddington, eds.), Cardiff Studies in Archaeology, Oxbow Books, Oxford: 99-118.

Marginedas, F.; Rodríguez-Hidalgo, A.; Soto, M.; Bello, S.M.; Cáceres, I.; Huguet, R.; Saladié, P. (2020): Making skull cups: Butchering traces on cannibalized human skulls from five European archaeological sites. Journal of Archaeological Science, 114: 105076. https://doi.org/10.1016/j.jas.2020.105076

Marc, A.T. (2016): "Mortuary practices in the Wietenberg Culture from Transylvania". Funerary Practices During the Bronze and Iron Ages in Central and Southeast Europe (V. Sîrbu; M. Jevtić; K. Dmitrović; M. Ljuština, eds.), Faculty of Philosophy /National Museum, Belgrade-Čačak: 53-73.

Marinas Díez, E. (2014): El yacimiento de "La Dehesa". Una aproximación al mundo funerario Protocogotas. Madrid: Ed. MArqAUDEMA. Serie Antropología.

Martínez Calvo, V.; Moreno García, E.; Gallego Esquinas, C.; López Jiménez, O. (2015): El yacimiento inédito de El Juncal (Getafe). Primera aproximación. En La Arqueología en el trazado ferroviario del sur de la comunidad de Madrid. Una aventura fascinante. ADIF, Madrid: 247-276.

Misiego Tejeda, J.C.; Pérez Rodríguez, F.J.; Sanz García, F.J.; Marcos Contreras, G.J.; Martín Carbajo, M.A. (1992): La Huelga (Dueñas, Palencia). Un yacimiento del Bronce Medio en la Meseta Norte. Revista de Arqueología, 136: 18-25.

Moraitis, K.; Eliopoulos, C.; Spiliopoulou, C. (2008): Fracture Characteristics of Perimortem Trauma in Skeletal Material. The Internet Journal of Biological Anthropology 3(2). http://ispub.com/ IJBA/3/2/11380.

Moral del Hoyo, S.; Rodríguez Marcos, J.A.; Díez Fernández-Lomana, J.C. (2003-4): Las cerámicas de la Cueva de El Mirador (Ibeas de Juarros, Burgos) en el contexto del Bronce Medio de la Submeseta norte. Boletín del Seminario de Estudios de Arte y Arqueología, 69-70: 57-74.

Olalde, I.; Mallick, S.; Patterson, N. ...... et al. (2019): "The genomic history of the Iberian Peninsula over the past 8000 years". Science, 363 (6432): 1230-1234 (https://doi.org/10.1126/science.aav4040). Incluye Supplementary Materials. www.sciencemag.org/content/363/6432/1230/supp1/DC1

Palol, P. de; Wattenberg, F. (1974): Carta Arqueológica de España. Valladolid. Diputación Provincial, Valladolid.

Palomo Díez, S. (2015): Caracterización genética de las poblaciones de las Edades del Cobre y del Bronce de la Submeseta norte de la Península Ibérica. Tesis doctoral defendida en la Universidad Complutense de Madrid (disponible en https://eprints.ucm.es/34256/).

Patrimonio Inteligente (2019): Trabajos de documentación arqueológica para el desarrollo del proyecto de parque eólico "Carrecastro", TT.MM. de Tordesillas y Velilla (Valladolid). Informe Técnico. Informe técnico depositado en el Servicio Territorial de Cultura de la Junta de Castilla y León. Valladolid. 
Pérez Rodríguez, F.J.; Misiego Tejeda, J.C.; Sanz García, F.J.; Marcos Contreras, G.J.; Martín Carbajo, M.A.; Fernández Giménez, J.M. (1994): "La Huelga": un interesante yacimiento de la Edad del Bronce en el centro de la Cuenca del Duero (Dueñas, Palencia). Numantia, 5: 11-32.

Pettitt, P. (2018): Hominin evolutionary thanatology from the mortuary to funerary realm: the palaeoanthropological bridge between chemistry and culture. Philosophical Transactions of the Royal Society B: Biological Sciences, 373: 20180212. http://dx.doi.org/10.1098/rstb.2018.0212

Poulain, T.; Lange, G. (1984): Le camp chasséen de Jonquières (Oise): V. les vestiges humains. Revue archéologique de Picardie, 1-2: 265-267.

Redfern, R. (2008): New evidence for Iron Age secondary burial practice and bone modification from Gussage All Saints and Maiden Castle (Dorset, England). Oxford Journal of Archaeology, 27(3): 281301.

Reimer, P. J.; Bard, E.; Bayliss, A.; Beck, J. W.; Blackwell, P. G.; Bronk Ramsey, C.; van der Plicht, J. (2013). IntCal13 and Marine13 Radiocarbon Age Calibration Curves 0-50,000 years cal BP. Radiocarbon, 55: 1869-1887. https://doi.org/10.2458/azu_js_rc.55.16947

Rojo, M.A.; Peña, L.; Royo, J.I.; Tejedor, C.; García, I.; Arcusa, H.; Garrido, R.; Moreno, M.; Mazzuco, N.; Gibaja, J.F.; Ortega, D.; Kromer, B.; Alt, K.W. (2013): Pastores trashumantes del Neolítico antiguo en un entorno de alta montaña: secuencia crono-cultural de la Cova de Els Trocs, San Feliú de Veri (Huesca). Boletín del Seminario de Arte y Arqueología, 79: 9-55.

Rojo, M.; Royo, J.I.; Garrido, R.; García, I.; Tejedor, C.; Arcusa, H.; Peña, L. y Moreno, M. (2015): La Cueva de Els Trocs: un asentamiento del Neolítico antiguo junto al Pirineo axial. En V.S. Gonçalves, M. Diniz y A. Catarina Sousa (eds.): V Congresso do Neolítico Peninsular. Lisboa, 7-9 abril de 2011. Centro de Arqueologia da Univesidade de Lisboa, Estudo y Memorias 8. Lisboa: $189-198$.

Rousseau, E. (2012): Pratique des têtes coupées chez les Gaulois: les données archéologiques. Crânes trophées, crânes d'ancêtres et autres pratiques autour de la tête (B. Boulestin; D. Henry-Gambier, dir.), Archaeopress, Oxford: 117-138. [BAR Int. Ser. 2415].

Saladié, P.; Rodríguez-Hidalgo, A. (2017): Archaeological Evidence for Cannibalism in Prehistoric Western Europe: from Homo antecessor to the Bronze Age. Journal of Archaeological Method and Theory, 24: 1034-1071. https://doi.org/10.1007/s10816-016-9306-y

Sánchez Polo, A. (2010): La muerte en arqueología: visiones cruzadas / posiciones encontradas. El Futuro del Pasado, 1: 173-187.

Santana, J.; Velasco, J.; Ibáñez, J.J.; Braemer, F. (2012): Crania with mutilated facial skeletons: A new ritual treatment in an early Pre-Pottery Neolithic B cranial cache at Tell Qarassa North (South Syria). American Journal of Physical Anthropology 149(2): 205-216. http://dx.doi.org/10.1002/ ajpa.22111

Santana, J.; Velasco, J.; Balbo, A.; Iriarte, E.; Zapata, L.; Teira, L.; Nicolle, C.; Braemer, F.; Ibáñez, J. (2015): Interpreting a ritual funerary area at the Early Neolithic site of Tell Qarassa North (South Syria, late 9th millennium BC). Journal of Anthropological Archaeology, 37: 112-127. http://dx.doi.org/10.1016/j. jaa.2014.12.003

Santana, J.; Rodríguez-Santos, F. J.; Camalich-Massieu, Ma D.; Martín-Socas, D.; Fregel, R. (2019): Aggressive or funerary cannibalism? Skull-cup and human bone manipulation in Cueva de El Toro (Early Neolithic, southern Iberia). American Journal of Physical Anthropology, 169(1):31-54. https:// doi.org/10.1002/ajpa.23805

Schmitt, A. (2017): Middle Neolithic burials in Mediterranean France: honouring or rejecting the dead?. West \& East, II: 63-82. https://doi.org/10.13137/2499-7331/15059

Sorg, M.H. (2019): Differentiating trauma from taphonomic alterations. Forensic Science International, 302: 109893. https://doi.org/10.1016/j.forsciint.2019.109893

Spencer, S.D. (2012): Detecting violence in the archaeological record: Clarifying the timing of trauma and manner of death in cases of cranial blunt force trauma among pre-Columbian Amerindians of WestCentral Illinois. International Journal of Paleopathology, 2(2-3): 112-122. https://doi.org/10.1016/j. ijpp.2012.09.007

Strato S.L. (1992): Excavación arqueológica en el yacimiento de "La Huelga" (Dueñas, Palencia) Parcela $n^{o}$ 45, Polígono de Dueñas (IRYDA) Propiedad Áridos García. Segunda actuación arqueológica. Agosto 1992. Informe técnico depositado en el Servicio Territorial de Cultura de la Junta de Castilla y León. Palencia. 
Szécsényi-Nagy, A.; Roth, C.; Brandt, G. et al. (2017): The maternal genetic make-up of the Iberian Peninsula between the Neolithic and the Early Bronze Age. Scientific Reports, 7: $15644 \mathrm{https} / / /$ doi. org/10.1038/s41598-017-15480-9

Ucko, P.J. (1969): Ethnography and Archaeological Interpretation of Funerary Remains. World Archaeology, 1(2): 262-280.

Valera, A.C. (2019): Fragmentation and Depositions in Pre and Proto-Historic Portugal. ERAArqueologia, Lisboa.

Velasco Vázquez, J.; Delgado Darías, T. (2005): Apéndice I. Consideraciones preliminares sobre los restos humanos de la Cueva de La Revilla (Atapuerca, Burgos). En Abarquero et al.: 128-136.

Velasco Vázquez, J.; Esparza Arroyo, A. (2016): Muertes ritualizadas en la Edad del Bronce de la Península Ibérica: un enterramiento inusual en Los Rompizales (Quintanadueñas, Burgos). Munibe AntropologiaArkeologia, 67: 75-103. https://www.doi.org/10.21630/maa.2016.67.04

Vergés, J. M.; Allué, E.; Angelucci, D. E.; Cebrià, A.; Díez, C.; Fontanals, M.; Manyanos, A.; Montero, S.; Moral, S.; Vaquero, M.; Zaragoza, J. (2002): La Sierra de Atapuerca durant el Holoceno: datos preliminares sobre las ocupaciones de la Edad del Bronce en la Cueva del Mirador (Ibeas de Juarros, Burgos). Trabajos de Prehistoria, 59(1): 107-126.

Wattenberg, F. (1957): Hallazgos arqueológicos en Renedo de Esgueva (Valladolid). Boletín del Seminario de Estudios de Arte y Arqueología, XXIII: 189-191.

Wedel, V. L.; Galloway, A. (2013): Broken bones: anthropological analysis of blunt force trauma. Springfield: Charles C Thomas.

Wheatley, B.P. (2008): Perimortem or postmortem bone fractures? An experimental study of fracture patterns in deer femora. Journal of Forensic Science, 53(1): 69-72. https://doi.org/10.1111/j.15564029.2008.00593.x

Zammit, J. (2003): Pratiques mortuaires et traitement du cadavre ancien (plaidoyer pour une nécroarchéologie). Revue Archéologique de Picardie, 21: 185-194. 